\title{
Asymptotic symmetries of electromagnetism at spatial infinity
}

\author{
Marc Henneaux ${ }^{a, b}$ and Cédric Troessaert ${ }^{c}$ \\ ${ }^{a}$ Université Libre de Bruxelles and International Solvay Institutes, \\ ULB-Campus Plaine CP231, B-1050 Brussels, Belgium \\ ${ }^{b}$ Collège de France, \\ 11 place Marcelin Berthelot, 75005 Paris, France \\ ${ }^{c}$ Max-Planck-Institut für Gravitationsphysik (Albert-Einstein-Institut), \\ Am Mühlenberg 1, DE-14476 Potsdam, Germany \\ E-mail: henneaux@ulb.ac.be, ctroessaert@aei.mpg.de
}

ABSTRACT: We analyse the asymptotic symmetries of Maxwell theory at spatial infinity through the Hamiltonian formalism. Precise, consistent boundary conditions are explicitly given and shown to be invariant under asymptotic angle-dependent $u(1)$-gauge transformations. These symmetries generically have non-vanishing charges. The algebra of the canonical generators of this infinite-dimensional symmetry with the Poincaré charges is computed. The treatment requires the addition of surface degrees of freedom at infinity and a modification of the standard symplectic form by surface terms. We extend the general formulation of well-defined generators and Hamiltonian vector fields to encompass such boundary modifications of the symplectic structure. Our study covers magnetic monopoles.

KeYwords: Gauge Symmetry, Global Symmetries, Space-Time Symmetries

ARXiv EPRINT: 1803.10194 


\section{Contents}

1 Introduction 1

2 Some background 3

2.1 Action and gauge symmetries 3

2.2 Poincaré transformations 5

2.3 Liénard-Wiechert solution $\quad 7$

2.4 Magnetic monopoles $\quad 7$

3 How to make the symplectic form finite $\quad 8$

3.1 Condition on the radial components 8

$\begin{array}{ll}3.2 & \text { Conditions on the angular components }\end{array}$

4 Asymptotic analysis: I. No magnetic charge 10

$\begin{array}{lll}4.1 & \text { Generalization of the boundary conditions } & 10\end{array}$

4.2 Lorentz boosts: need to add a boundary term to the symplectic structure 11

$\begin{array}{lll}4.3 & \text { Complete action } & 13\end{array}$

4.4 New global symmetry - alternative form of the action 13

$\begin{array}{ll}4.5 \text { Poincaré charges } & 15\end{array}$

$\begin{array}{lll}5 & \text { Asymptotic analysis: II. Magnetic charges } & 17\end{array}$

6 Conclusions 18

$\begin{array}{ll}\text { A Poisson structure } & 20\end{array}$

B Electromagnetism in hyperbolic slicings $\quad 21$

B.1 Connection with previous work 21

B.2 Asymptotic behaviour of the fields as one goes to null infinity 23

C Explicit large gauge transformations and link with null infinity 24

\section{Introduction}

Recent work on the asymptotic structure of electromagnetism in Minkowski spacetime has revealed the remarkable presence of an infinite-dimensional symmetry at infinity, which enables one to view the soft photon theorems in quantum electrodynamics as the associated Ward identities [1-11] (for a review of these fascinating developments, see [12]).

Most of these studies were carried out at null infinity. The purpose of this paper is to shed light on these questions by performing the analysis at spatial infinity. A description 
of the symmetry and its associated charges on standard spacelike hyperplanes adapted to inertial Lorentz observers, who have access to the information available at null infinity since their hyperplanes of simultaneity are Cauchy surfaces, must indeed be possible and is in fact so, as we shall show. Our work also resolves the tension between the absence of effective infinite symmetry at spatial infinity found in some earlier work and the recent developments mentioned above.

In order to perform the analysis at spacelike infinity, one must provide there precise boundary conditions on the dynamical variables. These conditions should be consistent, i.e., should fulfill the following criteria:

- They should make the action, and in particular the symplectic form, finite.

- They should be such that all Poincaré transformations are symmetries of the theory. That is, the Poincaré transformations, including the dynamical ones (time translations and Lorentz boosts), should be canonical transformations that leave the boundary conditions invariant and have well-defined (finite) canonical generators.

- They should include the "physically" relevant solutions (in particular the Coulomb potential for a charge at rest).

Boundary conditions fulfilling these criteria were given in [13]. These boundary conditions involve parity conditions inspired by [14] and are invariant under an infinitedimensional set of angle dependent $u(1)$ transformations fulfilling also some definite parity conditions. However, the corresponding charges turn out to vanish for all configurations obeying the boundary conditions, indicating that they are "proper gauge transformations" [15] not changing the physical state of the system. This shows that the analysis of [13] must be completed and that the problem of finding consistent boundary conditions allowing for a non trivial action of the asymptotic infinite-dimensional symmetries requires delicate investigation.

A similar problem arises for pure gravity, where the BMS group [16-23] investigated at null infinity is absent with the parity conditions of [14]. The difficulty was solved in [24] where new boundary conditions involving a crucial "twist" of the parity conditions for the angular components of the fields were proposed and shown to yield a non trivial action of the BMS group.

We show in this paper that a similar twist with respect to the parity conditions of [13] is necessary in the electromagnetic case. We also prove that it is in fact sufficient that this twist be an improper gauge transformation for the emergence of the non-trivial infinitedimensional symmetry. What is crucial is to allow non zero twisted parity components of the angular components of the potential. The virtue of taking these twisted components to be simply an improper gauge transformation and not arbitrary functions with twisted parity, is that one can then easily cover magnetic sources. Furthermore, this restricted form of the twist eliminates singular behaviour of some of the fields as one tends to null infinity.

We also investigate Lorentz invariance, known to be a subtle issue due to the longrange features of the electromagnetic field [25, 26], already at the classical level (see e.g. [27] and [1] and references therein). The most unexpected feature coming out of our analysis 
is the manner in which the Lorentz boosts are canonically realized. Indeed, these fail to be canonical transformations, unless one adds a surface degree of freedom at infinity. This degree of freedom contributes to the symplectic structure, which acquires therefore an extra surface term. Boundary contributions to the symplectic form were discussed earlier in [28] in the different context of isolated horizons. Here, our extra surface term in the symplectic structure reproduces the surface contribution of [11] when one fixes the gauge by relating the temporal component $A_{0}$ of the vector potential to the new surface degree of freedom. However, we prefer not to fix the gauge in our general derivation and leave $A_{0}$ unrelated to the surface degree of freedom. Once the new degree of freedom and the surface contribution have been included, the Lorentz group is canonically realized.

The idea of introducing surface degrees of freedom was pursued previously in [29-32] to describe the infrared sector of gauge theories, and more recently in [33] where they appear as holographic Stückelberg fields, but we have not explored the connection (if any) between the variables introduced there and the variables introduced here.

Our paper is organized as follows. The next section, section 2, recalls some background information on electromagnetism in Minkowski space. The explicit action of the Poincaré group on the leading orders of the asymptotic fields is given and the asymptotic form of some key solutions (Liénard-Wichiert potentials, magnetic monopoles) is given. We then show in section 3 that the finiteness of the bulk symplectic form imposes extra conditions on the asymptotic fields. Combined with the known behaviour of the key solutions, this leads to a definite set of asymptotic conditions. The asymptotic behaviour involves a twist of the parity conditions for the angular part of the fields, which is crucial for the emergence of a non trivial infinite-dimensional asymptotic symmetry. Section 4 constitutes the core of our paper. It deals with the zero magnetic charge case, which illustrates all the main points. We first exhibit the need to modify the symplectic form by boundary contributions which involve new surface degrees of freedom in order for the boosts to be canonical transformations. We then provide the complete action, with these new degrees of freedom included. The global symmetries are worked out and shown to involve an angle-dependent $u(1)$ algebra. The treatment is extended in section 5 to cover magnetic sources. The extension is direct. Indeed, all the new conceptual points are already present in the zero magnetic charge case of section 4 since they are related to the twist in the parity conditions. Section 6 is devoted to concluding comments and open questions. In particular, the way to cover Taub-NUT and magnetic charges in the Einstein-Maxwell system is indicated. Finally, appendix A extends the general formulation of well-defined generators and Hamiltonian vector fields to cover boundary modifications of the symplectic structure, while appendices $\mathrm{B}$ and $\mathrm{C}$ provide the explicit link between the asymptotic symmetry algebras at null infinity and spatial infinity, and demonstrate their equality.

\section{Some background}

\subsection{Action and gauge symmetries}

We start with free electromagnetism in Minkowski space, in standard Minkowskian coordinates. The dynamical variables to be varied in the action are the spatial components 
$A_{i}$ of the vector potential, their conjugate momenta $\pi^{i}$ (equal to the electric field) and the temporal component $A_{0} \equiv A_{t}$ of the vector potential which plays the role of Lagrange multiplier for the constraint

$$
\mathcal{G}=-\partial_{i} \pi^{i} \approx 0
$$

(Gauss' law). We use the symbol $\approx$ to denote equality on the constraint's surface. The action is

$$
S_{H}\left[A_{i}, \pi^{i}, A_{0}\right]=\int d t\left\{\int d^{3} x \pi^{i} \partial_{t} A_{i}-\int d^{3} x\left(\frac{1}{2} \pi^{i} \pi_{i}+\frac{1}{4} F^{i j} F_{i j}+A_{t} \mathcal{G}\right)+F_{\infty}\right\}
$$

where $F_{\infty}$ is a surface term at spatial infinity $(r \rightarrow \infty)$, which depends on the boundary conditions and which will be discussed below.

One could couple charges (massive or massless) to the electromagnetic field but we can assume that their fields decay sufficiently fast at spatial infinity that they do not directly contribute to the surface integrals. They do, however, indirectly contribute by changing the fluxes. For instance, if Gauss' law with zero right hand side holds everywhere in space $\left(\simeq \mathbb{R}^{3}\right)$, the flux of the electric field at spatial infinity vanishes. The presence of charges replaces $\mathcal{G} \equiv-\partial_{i} \pi^{i} \approx 0$ by $\mathcal{G} \equiv-\partial_{i} \pi^{i}+j^{0} \approx 0$ where $j^{0}$ is the charge density, and modifies the flux of the electric field at infinity, which is no longer zero. Assuming that the electric flux at infinity is non zero is the way we shall take the charges into account.

The electromagnetic field and its conjugate are usually taken to possess the following decay at spatial infinity,

$$
A_{i}=\frac{1}{r} \bar{A}_{i}+\frac{1}{r^{2}} A_{i}^{(1)}+o\left(r^{-2}\right), \quad \pi^{i}=\frac{1}{r^{2}} \bar{\pi}^{i}+\frac{1}{r^{3}} \pi^{(1) i}+o\left(r^{-3}\right)
$$

where the coefficients of the various powers of $r^{-1}$ are arbitrary functions on the 2-sphere, i.e., of the angles $x^{A}$ used to parametrize it. ${ }^{1}$ We shall find it necessary to strengthen (2.3). Extra constraints are indeed needed since the conditions (2.3) by themselves do not guarantee finiteness of the symplectic structure. So, the fall-off conditions (2.3) are not the final boundary conditions. The form of the extra constraints will be explicitly described in section 3 below.

The boundary conditions (2.3) are invariant under gauge transformations generated by the first-class constraint-generator $\mathcal{G}$ :

$$
\delta_{\epsilon} A_{i}=\partial_{i} \epsilon, \quad \delta_{\epsilon} \pi^{i}=0,
$$

provided the gauge parameter $\epsilon$ has the asymptotic behaviour

$$
\epsilon=\bar{\epsilon}\left(x^{A}\right)+\frac{1}{r} \epsilon^{(1)}\left(x^{A}\right)+o\left(r^{-1}\right) .
$$

Further conditions limiting the functional class to which $\epsilon$ belongs will of course appear when strengthening the boundary conditions (2.3). The generator of (2.4) is explicitly

$$
G[\epsilon]=\int d^{3} x \epsilon \mathcal{G}+\oint d^{2} S_{i} \bar{\epsilon} \bar{\pi}^{i} \approx \oint d^{2} S_{i} \bar{\epsilon} \bar{\pi}^{i}
$$

\footnotetext{
${ }^{1}$ We shall assume "uniform smoothness" [18] whenever needed, i.e., $\partial_{r} o\left(r^{-k}\right)=o\left(r^{-k-1}\right), \partial_{A} o\left(r^{-k}\right)=$ $o\left(r^{-k}\right)$.
} 
where the surface term is determined by the methods of [14]. The electric charge is associated with the improper gauge transformation generated by $\epsilon=1$. We recall that an "improper" gauge transformation [15] changes the physical state of the system and has non-vanishing charge. It should not be quotiented out.

To complete the description of the asymptotic behaviour, we need to specify the fall-off of the Lagrange multiplier $A_{t}$. Since $A_{t}$ parametrizes the gauge transformation performed in the course of the evolution, we take for $A_{t}$ the same fall-off as for the gauge parameter $\epsilon$,

$$
A_{t}=\bar{A}_{t}\left(x^{A}\right)+\frac{1}{r} A_{t}^{(1)}\left(x^{A}\right)+o\left(r^{-1}\right) .
$$

If $\bar{A}_{t}\left(x^{A}\right)=C \neq 0$, the time evolution involves a non-trivial improper gauge transformation. The term $\bar{A}_{t}\left(x^{A}\right)$ will be subject to the same extra conditions as the gauge parameter $\epsilon$ when strengthening the boundary conditions.

\subsection{Poincaré transformations}

We now turn to Poincaré transformations. A general deformation of a spacelike hyperplane can be decomposed into normal and tangential components, denoted by $\xi$ and $\xi^{i}$, respectively. A general Poincaré transformation corresponds to the deformation

$$
\begin{aligned}
\xi & =b_{i} x^{i}+a^{\perp} \\
\xi^{i} & =b^{i}{ }_{j} x^{j}+a^{i}
\end{aligned}
$$

where $b_{i}, b_{i j}=-b_{j i}, a^{\perp}$ and $a^{i}$ are arbitrary constants. The constants $b_{i}$ parametrize the Lorentz boosts, whereas the antisymmetric constants $b_{i j}=-b_{j i}$ parametrize the spatial rotations. The constants $a^{\perp}$ and $a^{i}$ are standard translations.

Under such a deformation, the fields transform as

$$
\begin{aligned}
\delta A_{i} & =\xi \pi_{i}+\xi^{j} F_{j i}+\partial_{i} \zeta \\
\delta \pi^{i} & =\partial_{m}\left(F^{m i} \xi\right)+\partial_{m}\left(\xi^{m} \pi^{i}\right)-\left(\partial_{m} \xi^{i}\right) \pi^{m}-\xi^{i} \partial_{m} \pi^{m} .
\end{aligned}
$$

The transformation of the fields is really defined up to a gauge transformation. This is the reason why we have included the term $\partial_{i} \zeta$ in the transformation of $A_{i}$. A definite choice of accompanying gauge transformation will be made below to get simple expressions for the algebra. It is clear that the fall-off (2.3) is preserved under these transformations provided $\zeta$ behaves as in $(2.5)$.

For later purposes, we rewrite the boundary conditions in spherical coordinates. One gets, recalling that the momenta carry a unit density weight:

$$
\begin{array}{rlrl}
A_{r} & =\frac{1}{r} \bar{A}_{r}+\frac{1}{r^{2}} A_{r}^{(1)}+o\left(r^{-2}\right), & \pi^{r} & =\bar{\pi}^{r}+\frac{1}{r} \pi^{(1) r}+o\left(r^{-1}\right), \\
A_{A} & =\bar{A}_{A}+\frac{1}{r} A_{A}^{(1)}+o\left(r^{-1}\right), & \pi^{A}=\frac{1}{r} \bar{\pi}^{A}+\frac{1}{r^{2}} \pi^{(1) A}+o\left(r^{-2}\right),
\end{array}
$$

while the form of $A_{t}$ remains unchanged as it is a spatial scalar. The coordinates $x^{A}$ are coordinates on the two-sphere. We will also need the Poincare transformations of the 
leading orders, which are invariant under translations and transform only under boosts and spatial rotations. Recalling that the above transformations read $\delta A_{i}=\frac{\xi \pi_{i}}{\sqrt{g}}+\xi^{j} F_{j i}+\partial_{i} \zeta$ and $\delta \pi^{i}=\partial_{m}\left(\sqrt{g} F^{m i} \xi\right)+\partial_{m}\left(\xi^{m} \pi^{i}\right)-\left(\partial_{m} \xi^{i}\right) \pi^{m}-\xi^{i} \partial_{m} \pi^{m}$ in general curvilinear coordinates, where $g_{i j}$ is the flat metric in those coordinates, one finds explicitly

$$
\begin{aligned}
\delta_{b, Y} \bar{A}_{r}= & \frac{b}{\sqrt{\bar{\gamma}}} \bar{\pi}^{r}+Y^{A} \partial_{A} \bar{A}_{r}, \\
\delta_{b, Y} \bar{A}_{A}= & \frac{b}{\sqrt{\bar{\gamma}}} \bar{\gamma}_{A B} \bar{\pi}^{B}+Y^{B}\left(\partial_{B} \bar{A}_{A}-\partial_{A} \bar{A}_{B}\right)+\partial_{A} \bar{\zeta}, \\
\delta_{b, Y} \bar{\pi}^{r}= & \sqrt{\bar{\gamma}} \bar{D}^{A}\left(b \partial_{A} \bar{A}_{r}\right)+\partial_{A}\left(Y^{A} \bar{\pi}^{r}\right), \\
\delta_{b, Y} \bar{\pi}^{A}= & \sqrt{\bar{\gamma}} \bar{D}_{B}\left(b \bar{\gamma}^{B C} \bar{\gamma}^{A D}\left(\partial_{C} \bar{A}_{D}-\partial_{D} \bar{A}_{C}\right)\right) \\
& +\partial_{B}\left(Y^{B} \bar{\pi}^{A}\right)-\partial_{B} Y^{A} \bar{\pi}^{B}-Y^{A} \partial_{B} \bar{\pi}^{B}
\end{aligned}
$$

where we have set, in terms of the unit metric $\bar{\gamma}_{A B}$ on the sphere,

$$
g_{A B}=r^{2} \bar{\gamma}_{A B}
$$

and

$$
\begin{aligned}
& \xi=r b+T, \quad \xi^{r}=W, \quad \xi^{A}=Y^{A}+\frac{1}{r} \bar{D}^{A} W, \\
& \bar{D}_{A} \bar{D}_{B} W+\bar{\gamma}_{A B} W=0, \quad \bar{D}_{A} \bar{D}_{B} b+\bar{\gamma}_{A B} b=0, \quad \mathcal{L}_{Y} \bar{\gamma}_{A B}=0, \quad \partial_{A} T=0 .
\end{aligned}
$$

The quantities $b, Y^{A}, T$ and $W$ are functions on the sphere. The first two, $b$ and $Y^{A}$, describe the homogeneous Lorentz transformations, while $T$ and $W$, which do not appear in the transformation laws (2.14)-(2.17) of the leading orders, describe the translations. Explicitly,

$$
\begin{aligned}
b & =b_{1} \sin \theta \cos \varphi+b_{2} \sin \theta \sin \varphi+b_{3} \cos \theta \\
Y & =m^{1}\left(-\sin \varphi \frac{\partial}{\partial \theta}-\frac{\cos \theta}{\sin \theta} \cos \varphi \frac{\partial}{\partial \varphi}\right)+m^{2}\left(\cos \varphi \frac{\partial}{\partial \theta}-\frac{\cos \theta}{\sin \theta} \sin \varphi \frac{\partial}{\partial \varphi}\right)+m^{3} \frac{\partial}{\partial \varphi}
\end{aligned}
$$

and

$$
W=a^{1} \sin \theta \cos \varphi+a^{2} \sin \theta \sin \varphi+a^{3} \cos \theta .
$$

Finally, $\bar{D}_{A}$ is the covariant derivative associated with $\bar{\gamma}_{A B}$ and $\bar{D}^{A}=\bar{\gamma}^{A B} \bar{D}_{B}$.

The transformation laws (2.14)-(2.17) of the leading orders possess interesting features:

- They do not mix radial and angular components. The radial variables transform among themselves and so do also the angular ones. This implies that one can treat independently the radial and angular components in the boundary conditions.

- Since $b$ and $Y^{A}$ are odd under the parity transformation $x^{i} \rightarrow-x^{i}$, which we formally write as $x^{A} \rightarrow-x^{A}$ in terms of the coordinates on the sphere, ${ }^{2}$ one can consistently

\footnotetext{
${ }^{2}$ Note that in terms of standard spherical coordinates, the antipodal map is actually $\theta \rightarrow \pi-\theta$ and $\varphi \rightarrow \varphi+\pi$ (and $r \rightarrow r$ ). This implies $d \theta \rightarrow-d \theta$ and $d \varphi \rightarrow d \varphi$. Therefore, the condition that $A_{A}$ is even (for example), i.e., $A_{A}\left(-x^{B}\right)=A_{A}\left(x^{B}\right)$, which really means that the one-form $A=A_{A} d x^{A}$ is odd (i.e., $\Phi^{*} A=-A$, where $\Phi^{*}$ denotes the pullback by the antipodal map), is equivalent to the statement that $A_{\theta}$ is even and $A_{\varphi}$ is odd. Similar considerations apply to the odd case $A_{A}\left(-x^{B}\right)=-A_{A}\left(x^{B}\right)$.
} 
impose parity conditions on the leading orders of the canonical variables. That is, one can require the components of the potential to have a definite parity and their conjugate momenta to have the opposite one. This is preserved by the Lorentz transformations (if one chooses the gauge transformation $\zeta$ appropriately). Furthermore, one can consider different parity conditions for the radial and angular components since they transform independently.

In polar coordinates, the conserved charge (2.6) takes the form

$$
G[\epsilon] \approx \oint d^{2} x \bar{\epsilon} \bar{\pi}^{r}
$$

It follows from our boundary conditions that $\bar{A}_{r}$ is gauge invariant since $\partial_{r} \epsilon=O\left(r^{-2}\right)$. The gauge invariance of $\bar{A}_{r}$ can be phrased in familiar terms by observing that the radial integral (along any fixed ray) $\int_{R}^{2 R} d r A_{r}$ transforms as $\epsilon(2 R)-\epsilon(R)=O\left(\frac{1}{R}\right)$ and is thus gauge invariant in the limit $R \rightarrow \infty$. This integral is equal to $\bar{A}_{r} \ln 2$ in that limit. The symmetry generated by $\bar{A}_{r}$ will be clarified below.

\subsection{Liénard-Wiechert solution}

To motivate the boundary conditions below, we now consider various solutions. We start with the Coulomb potential.

The Coulomb solution has $\bar{A}_{r}=0, \bar{\pi}^{r}=\sin \theta, \bar{A}_{A}=0$ and $\bar{\pi}^{A}=0$. By boosting it, one generates the Liénard-Wiechert solution. Since $\bar{\pi}^{r}$ is even, the Liénard-Wiechert solution is characterized by the following parities of the radial components,

$$
\bar{A}_{r}\left(-x^{A}\right)=-\bar{A}_{r}\left(x^{A}\right), \quad \bar{\pi}^{r}\left(-x^{A}\right)=\bar{\pi}^{r}\left(x^{A}\right) .
$$

The statement that $\bar{A}_{r}$ is odd is gauge invariant because $\bar{A}_{r}$ is gauge invariant.

As to the angular components, both $\bar{\pi}^{A}$ and $\bar{F}_{A B}$ remain zero,

$$
\bar{\pi}^{A}=0, \quad \bar{F}_{A B}=0 .
$$

This implies that $\bar{A}_{A}$ reduces to a gauge transformation $\partial_{A} \bar{\Phi}$,

$$
\bar{A}_{A}=\partial_{A} \bar{\Phi}
$$

for some function $\bar{\Phi}$ of the angles. The value of $\bar{\Phi}$ depends on the choice of the accompanying gauge transformation $\zeta$ in (2.14)-(2.17). The choice $\zeta=0$ amounts to transform $A_{\mu}$ as $\xi^{\rho} F_{\rho \mu}$ under Poincaré transformations. One other possible choice is to take $\zeta$ so that the transformation of the potential $A_{\mu}$ is its Lie derivatives $\mathcal{L}_{\xi} A_{\mu}$ rather than $\xi^{\rho} F_{\rho \mu}$. The two choices differ by the gauge transformation $\partial_{\mu}\left(\xi^{\rho} A_{\rho}\right)$. The gauge parameter $\xi^{\rho} A_{\rho}$ is of order $O(1)$ for rotations and boosts.

\subsection{Magnetic monopoles}

For magnetic monopoles, the situation is somewhat reversed. The asymptotic Coulomb fields $\bar{A}_{r}$ and $\bar{\pi}^{r}$ vanish and remain zero under Poincaré transformations. The field of a monopole is purely angular and given by

$$
\pi^{A}=0, \quad F_{\theta \varphi}=\sin \theta
$$


when the monopole is at rest. It coincides with its leading order. The 2 -form $\frac{1}{2} \bar{F}_{A B} d x^{A} d x^{B}$ is odd, something that we write as $\bar{F}_{A B}\left(-x^{C}\right)=-\bar{F}_{A B}\left(x^{C}\right)$. By Poincaré transforming the monopole field, one generates $\bar{\pi}^{A}$ and $\bar{F}_{A B}$ that remain odd. The potential is not globally defined but on the sphere minus the two (antipodal) poles, one can take it to be even up to a gauge transformation, e.g., for the monopole at rest

$$
\bar{A}_{\theta}=\partial_{\theta} \bar{\Phi}, \quad \bar{A}_{\varphi}=-\cos \theta+\partial_{\varphi} \bar{\Phi}
$$

(see footnote 2 for conventions on parity terminology). The (not globally defined) gauge transformation that brings the monopole potential to the familiar form $\bar{A}_{\varphi}=(1-\cos \theta)$ regular at the North pole has gauge parameter $\epsilon=\varphi$ and is such that the one-form $d \epsilon=d \varphi$ is odd (opposite parity to that of $-\cos \theta d \varphi$ ).

\section{How to make the symplectic form finite}

Without further constraints, the symplectic potential derived from the bulk piece of $(2.2)$ is logarithmically divergent, since its dominant part is

$$
\int \frac{d r}{r} \int d^{2} x\left(\bar{\pi}^{r} \dot{\bar{A}}_{r}+\bar{\pi}^{A} \dot{\bar{A}}_{A}\right)
$$

To make it convergent, one must impose extra conditions on the leading components of the dynamical variables so that the integral on the 2 -sphere

$$
\int d^{2} x\left(\bar{\pi}^{r} \dot{\bar{A}}_{r}+\bar{\pi}^{A} \dot{\bar{A}}_{A}\right)
$$

vanishes.

We treat separately the radial and angular components.

\subsection{Condition on the radial components}

To make the symplectic form finite for the radial components, we impose a parity condition, as for gravity [14]. In view of our above discussion, we request

$$
\bar{A}_{r}\left(-x^{A}\right)=-\bar{A}_{r}\left(x^{A}\right), \quad \bar{\pi}^{r}\left(-x^{A}\right)=\bar{\pi}^{r}\left(x^{A}\right) .
$$

This agrees with [13]. As we have seen, this boundary condition contains the Coulomb field viewed in a moving frame. The antipodal symmetry of $\bar{\pi}^{r}$ is what remains of the spherical symmetry of the static field after an arbitrary Poincaré transformation has been performed. As we have also stressed, (3.3) is gauge invariant. The parity condition (3.3) will be imposed throughout the subsequent discussion.

Since $\bar{\pi}^{r}$ is even, the charges (2.24) reduce to

$$
G[\epsilon] \approx \oint d^{2} x \bar{\epsilon}_{\mathrm{even}} \bar{\pi}^{r}
$$

The odd part $\epsilon_{\text {odd }}$ of the gauge parameter $\epsilon$ gives a zero contribution to the charges $G[\epsilon]$. It follows that the gauge transformations with $\epsilon_{\text {odd }}$ are proper gauge transformations that do 
not change the physical state of the system. By contrast, the gauge transformations with $\epsilon_{\text {even }}$ are improper gauge transformations that do change the physical state of the system. The charge (3.4) is generically non zero.

These conclusions hold irrespectively of whatever extra conditions are imposed on the angular components of the fields to make $\int d^{2} x \bar{\pi}^{A} \dot{\bar{A}}_{A}$ vanish, conditions to which we now turn.

\subsection{Conditions on the angular components}

No magnetic charge. In the absence of magnetic charges, we impose the boundary conditions

$$
\bar{\pi}^{A}=0, \quad \bar{A}_{A}=\partial_{A} \bar{\Phi}
$$

for some function $\bar{\Phi}$ of the angles. As our previous discussion shows, these conditions are fullfilled by the Liénard-Wiechert potentials, and match therefore the setting adopted in most discussions of the behaviour of the fields at null infinity [12].

It might be tempting to set $\bar{\Phi}$ equal to zero by a gauge transformation but this would be illegitimate if the needed gauge transformation is improper, which is the case when $\bar{\Phi}$ is even. For that reason, we keep $\bar{\Phi}$ - or at least its even part. The odd part of $\bar{\Phi}$ defines instead a proper gauge transformation and can be set equal to zero if one so wishes, although it can be useful to keep it.

Note that the relevant part of $\bar{A}_{A}$ is odd since $\bar{\Phi}_{\text {improper }}$ is even. We could therefore assume that $\bar{A}_{A}$ is odd. This means that $\bar{A}_{r} d r$ and $\bar{A}_{A} d x^{A}$ have opposite parities, which is the analog of the "twisted parity conditions" considered in [24] for gravity.

Magnetic charges. In the presence of magnetic poles, we keep the same conditions on the radial components of the fields so as to allow electric sources, and we impose on the angular components the parity conditions

$$
\bar{A}_{A}=\bar{A}_{A}^{\text {even }}+\partial_{A} \bar{\Phi}, \quad \bar{\pi}^{A}\left(-x^{B}\right)=-\bar{\pi}^{A}\left(x^{B}\right)
$$

which are in agreement with the behaviour of the fields of magnetic sources. Here

$$
\bar{A}_{A}^{\text {even }}\left(-x^{B}\right)=\bar{A}_{A}^{\text {even }}\left(x^{B}\right) .
$$

Thus, the angular part of the potential is even up to a gauge transformation, while the angular part of the electric field is odd. Again, one cannot drop the gauge component $\partial_{A} \bar{\Phi}$ since its odd part defines an improper gauge transformation that changes the physical state. Thus both the even and odd parts of $\bar{A}_{A}$ are essential, and the odd part takes the form $\partial_{A} \bar{\Phi}$.

To make the symplectic form finite, i.e., $\int d^{2} x \bar{\pi}^{A} \dot{\bar{A}}_{A}$ vanish, we impose also the Poincaré invariant condition

$$
\partial_{A} \bar{\pi}^{A}=0 .
$$

Since $\partial_{r} \pi^{r}=O\left(r^{-2}\right)$, this condition is equivalent to demanding that Gauss' law holds asymptotically, i.e., that the leading order $O\left(r^{-1}\right)$ in $\partial_{i} \pi^{i}$ be absent. This restriction has no physical impact in the sense that it does not remove any solution, for which $\partial_{i} \pi^{i}$ 
is strictly zero. A similar condition was previously used for gravity in the study of the asymptotic symmetries of asymptotically $\mathrm{AdS}_{3}$ spacetimes in [34] and, more recently, in the hamiltonian analysis of $\mathrm{BMS}_{4}$ symmetries in [24].

It is clear that the action and the boundary conditions are invariant under $u(1)$ transformations that are even functions of the angles. This infinite-dimensional algebra acts non trivially. The boundary conditions of [13] assumed that $\bar{\Phi}$ was odd so that $\bar{A}_{A}$ was even. This choice corresponds to a uniform behaviour of the components of the vector potential and its conjugate in Cartesian coordinates, i.e., $\bar{A}_{i}=$ even, $\bar{\pi}^{i}=$ odd. It is the analog for electromagnetism of the boundary conditions of [14]. It freezes as we have seen the possibility of making improper gauge transformations and explains why the non trivial asymptotic symmetry was found to contain in that case only the global constant $u(1)$ transformations. The strict parity conditions $\bar{A}_{A}\left(-x^{B}\right)=\bar{A}_{A}\left(x^{B}\right)$ of [13] choose a definite point in the orbits of the angle-dependent $u(1)$ transformations. To be able to see the full orbits, one must allow an odd part in the potential, as in (3.6).

\section{Asymptotic analysis: I. No magnetic charge}

\subsection{Generalization of the boundary conditions}

We have exhibited so far an infinite-dimensional symmetry of electromagnetism characterized by an even function on the sphere. The emergence of the other half of the symmetry, characterized by an odd function, is rather subtle. The point is that the inclusion of an odd part in the vector potential, necessary as we have stressed to allow for the infinite symmetry, makes at the same time the action of the Lorentz transformations non canonical. One way to cure this problem is to modify the symplectic structure by a boundary term involving a new surface degree of freedom. The resulting action has a new symmetry involving the searched-for odd function on the sphere, which combines with the even function to yield the full symmetry displayed at null infinity.

The purpose of this section is to explain these somewhat unexpected features. We start with the case of no magnetic charge with the boundary conditions (3.5) on the angular variables. This case illustrates already all the main features of the general construction.

It is in fact interesting to consider more general boundary conditions than (3.5). We shall assume in this section that the angular part of the electric field $\bar{\pi}^{A}$ does not vanish but is an even function on the sphere that fulfills $\partial_{A} \bar{\pi}^{A}=0$, and that the angular part of the vector potential is an odd function of the sphere modulo a gradient, $\bar{A}_{A}\left(-x^{B}\right)=$ $-\bar{A}_{A}\left(x^{B}\right)+\partial_{A} \bar{\Phi}$. The even part of $\bar{\Phi}$ can actually be absorbed in a redefinition of $A_{A}$ so that we can assume $\bar{\Phi}$ to be odd. For that reason, one could drop $\bar{\Phi}$ since it defines a proper gauge transformation, but we choose to keep it as it simplifies some formulas. Thus, we take as boundary conditions in this section

$$
\begin{array}{cc}
\bar{A}_{A}\left(-x^{B}\right)=-\bar{A}_{A}\left(x^{B}\right)+\partial_{A} \bar{\Phi}, & \bar{\Phi}\left(-x^{B}\right)=-\bar{\Phi}\left(x^{B}\right), \\
\text { (Alternative - and not the final! - boundary conditions) } \\
\bar{\pi}^{A}\left(-x^{B}\right)=\bar{\pi}^{A}\left(x^{B}\right), & \partial_{A} \bar{\pi}^{A}=0 .
\end{array}
$$

These boundary conditions make the symplectic form finite. 
Because the angular components of the fields fulfill parity properties different from those of the radial components, one calls (4.1) and (4.2) "twisted parity conditions". These boundary conditions clearly contain (3.5) and hence, accommodate the Liénard-Wiechert potentials. They also allow for improper gauge transformations. We shall carry the analysis of the no-magnetic-charge case with these boundary conditions explaining along the way the simplifications that occur if $\bar{A}_{A}$ reduces to its pure (improper) gauge transformation piece and $\bar{\pi}^{A}$ vanishes as in (3.5).

There is an interest in carrying the analysis with the more general conditions (4.1) and (4.2) for various reasons. First, it is always a good policy to devise boundary conditions as flexible as possible. Second, the analysis of (4.1) and (4.2) does not cost much more work than the analysis of the conditions (3.5). Finally, the twisted parity conditions (4.1) and (4.2) are the analogs for electromagnetism of the twisted boundary conditions for gravity given in [24]. Their analysis provides therefore useful insight into the limitations and properties of these boundary conditions.

\subsection{Lorentz boosts: need to add a boundary term to the symplectic structure}

For the Poincaré group to be a symmetry of the theory, it should leave the action invariant. In particular, it should leave the symplectic structure invariant. There is no difficulty with the bulk terms since the Poincaré transformations have canonical bulk generators and are thus formally canonical transformations. The only subtlety comes from surface terms. These are usually neglected without actually checking that they are indeed zero. We shall show in this section that these terms are in fact not zero for all Poincaré transformations and need therefore special treatment.

The difficulty comes from the Lorentz boosts, to which we explicitly turn (the surface terms for the other Poincaré transformations can be easily verified to raise no problem as it will be explicitly checked in subsection 4.5 below).

The symplectic 2 -form derived from (2.2) is

$$
\Omega=\int d^{3} x d_{V} \pi^{i} d_{V} A_{i}
$$

where the product is the exterior product $\wedge$ of forms which we are not writing explicitly, and where we use the symbol $d_{V}$ for the exterior derivative in phase space in order not to introduce confusion with the spacetime exterior derivative.

The transformation defined by the vector field $X$ is canonical if $d_{V}\left(i_{X} \Omega\right)=0$. Evaluating this expression for the boosts $\xi=b r$, one finds

$$
d_{V}\left(i_{b} \Omega\right)=\int d^{3} x \partial_{m}\left(\sqrt{g} \xi d_{V} F^{m i}\right) d_{V} A_{i}
$$

where we have used $d_{V} \pi^{i} d_{V} \pi_{i}=0$. Integrating by parts and using $d_{V} F_{i j} d_{V} F^{i j}=0$, we get that $d_{V}\left(i_{b} \Omega\right)$ reduces to a surface term,

$$
d_{V}\left(i_{b} \Omega\right)=\oint d^{2} x \sqrt{g} \xi d_{V} F^{r i} d_{V} A_{i},
$$


an expression that can be transformed to

$$
\begin{aligned}
d_{V}\left(i_{b} \Omega\right) & =-\oint d^{2} x \sqrt{\bar{\gamma}} b d_{V} \bar{D}^{A} \bar{A}_{r} d_{V} \bar{A}_{A} \\
& =\oint d^{2} x \sqrt{\bar{\gamma}} d_{V} \bar{A}_{r} \bar{D}^{A}\left(b d_{V} \bar{A}_{A}\right)
\end{aligned}
$$

using the asymptotic form of the fields. This term would vanish if $\bar{A}_{A}$ was even, but in our case, $\bar{A}_{A}$ has a non trivial odd component. That odd component remains crucially present, and $d_{V}\left(i_{b} \Omega\right)$ does not vanish, even if it reduces to an improper gauge transformation, $\bar{A}_{A}=\partial_{A} \bar{\Phi}$ with $\bar{\Phi}$ even. Something must thus be done in order to accommodate Lorentz boosts.

One might try to impose a relationship between $\bar{A}_{r}$ and $\bar{A}_{A}$ so that the 2-form

$$
\oint d^{2} x \sqrt{\bar{\gamma}} b d_{V} \bar{D}^{A} \bar{A}_{r} d_{V} \bar{A}_{A}
$$

vanishes. This would be the analog of what was done in [35-37] when dealing with a slowly decaying scalar field in anti-de Sitter space. There is, however, no obvious relationship that can be imposed without at the same time destroying the asymptotic angle-dependent $u(1)$ at infinity, so that a different route must be followed.

We shall instead add a surface degree of freedom at infinity, which we denote by $\bar{\Psi}^{3}$ The field $\bar{\Psi}$ is at this stage a field living on the two-sphere at infinity, which can depend on time. To cancel the unwanted surface term (4.7), we add to the symplectic 2 -form the surface term

$$
-\oint d^{2} x \sqrt{\bar{\gamma}} d_{V} \bar{A}_{r} d_{V} \bar{\Psi}
$$

and postulate that $\bar{\Psi}$ transforms under boosts as

$$
\delta_{b} \bar{\Psi}=\bar{D}^{A}\left(b \bar{A}_{A}\right)+b \bar{A}_{r}
$$

The field $\bar{\Psi}$ could be restricted to be odd under parity, i.e., to be of the same parity as $\bar{A}_{r}$. We can however add to it an even part, which is clearly pure gauge since it drops from the surface term (4.8). Shifting $\bar{\Psi}$ by an arbitrary even function is then another proper gauge symmetry of the theory, which comes in addition to the proper gauge symmetries generated by $G[\epsilon]$ with $\epsilon$ odd. In other words, we take the field $\bar{\Psi}$ to be odd up to a proper gauge symmetry.

The second term in the right-hand side of (4.9) is even. It is therefore a pure gauge term, which we find convenient to add as it simplifies some formulas below. (When extended into the bulk, the transformation takes the convenient form (4.29).)

We also modify the transformation law of $A_{i}$ by adding a gauge transformation parametrized by the gauge parameter $\xi \Psi$, where $\Psi$ is any function that matches $\bar{\Psi}$ at

\footnotetext{
${ }^{3}$ The boundary field $\bar{\Psi}$ plays here a similar role to the extra boundary field $\phi_{ \pm}$introduced in [5] in order to complete the Poisson structure at future and past null infinity. Both fields parametrise improper gauge degrees of freedom. Using the results of appendix C, one can show that the fields $\phi_{ \pm}$defined at null infinity are linear combinations of $\bar{\Psi}$ and $\bar{\Phi}$.
} 
infinity as

$$
\Psi=\frac{\bar{\Psi}}{r}+\frac{\Psi^{(1)}}{r^{2}}+o\left(r^{-2}\right) .
$$

Which extension of $\bar{\Psi}$ one takes does not matter since two extensions will differ by a proper gauge transformation. The full transformation of $A_{i}$ under boosts is thus

$$
\delta A_{i}=\frac{1}{\sqrt{g}} \xi \pi_{i}+\partial_{i}(\xi \Psi)
$$

The extra gauge transformation does not yield extra term in the transformation of $\bar{A}_{r}$ $\left(\partial_{r}(b \Psi)\right.$ is of order $\left.r^{-2}\right)$, but does induce an extra surface term in the variation of the symplectic form equal to

$$
\oint d^{2} x b d_{V} \bar{\pi}^{r} d_{V} \bar{\Psi}
$$

With these transformations, the symplectic form is invariant and the boosts define canonical transformations. Note that the even part of $\bar{\Psi}$ in (4.11) defines a proper gauge transformation, while its odd part defines an improper gauge transformation.

\subsection{Complete action}

The action describing the dynamics with the new field $\bar{\Psi}$ included is

$$
\begin{aligned}
S_{H}\left[A_{i}, \pi^{i}, \bar{\Psi} ; A_{t}\right]=\int d t\{ & \int d^{3} x \pi^{i} \partial_{t} A_{i}-\int d^{3} x\left(\frac{1}{2 \sqrt{g}} \pi^{i} \pi_{i}+\frac{1}{4} \sqrt{g} F^{i j} F_{i j}+A_{t} \mathcal{G}\right) \\
& \left.-\oint d^{2} x \sqrt{\bar{\gamma}} \bar{A}_{r} \partial_{t} \bar{\Psi}\right\} .
\end{aligned}
$$

Variations of the extra boundary term in (4.13) give two extra equations of motion on the boundary:

$$
\partial_{t} \bar{A}_{r}=0, \quad \partial_{t} \bar{\Psi}=0 .
$$

The first one is not new as it is a consequence of the bulk equation of motion generated by $\pi^{r}$ :

$$
\partial_{t} A_{r}-\partial_{r} A_{t}-\frac{1}{\sqrt{g}} \pi_{r}=0 \quad \Rightarrow \quad \partial_{t} \bar{A}_{r}=0
$$

The second one is an equation of motion for the new surface degree of freedom $\bar{\Psi}$.

One could add to the action a surface Hamiltonian involving $\bar{A}_{r}$ that would modify the equation of motion for $\bar{\Psi}$. The choice made here, namely $H_{\text {Surface }}=0$, is the simplest one, and it is compatible with Poincaré invariance as discussed below.

\subsection{New global symmetry - alternative form of the action}

Note that the gauge generator (2.6) remains well-defined even after the symplectic structure has been modified because the leading term of the gauge parameter does not depend on the fields.

It is clear that the action is invariant under arbitrary shifts of $\bar{\Psi}$ which can depend on the angles,

$$
\delta_{\mu} \bar{\Psi}=\bar{\mu}, \quad \delta_{\mu} A_{i}=0, \quad \delta_{\mu} \pi^{i}=0, \quad \delta_{\mu} A_{t}=0
$$


The even part of the parameter $\mu$, which can have an arbitrary time-dependence, generates a pure gauge transformation, but the odd part, which must be time-independent in order to leave the action invariant, generates a global symmetry. The corresponding charge is $-\oint d^{2} x \sqrt{\bar{\gamma}} \bar{\mu} \bar{A}_{r}$ as we shall derive below, and is generically non-vanishing.

It turns out that both the even part of $\bar{\epsilon}$ and the odd part of $\bar{\mu}$ naturally combine into a single function. In order to make that structure manifest, it is useful to extend the surface degree of freedom $\bar{\Psi}$ into a dynamical bulk field $\Psi$, i.e., to treat (4.10) as a field to be varied in the action principle. This can be done provided one introduces at the same time the constraint that the conjugate $\pi_{\Psi}$ to $\Psi$ vanishes,

$$
\pi_{\Psi} \approx 0
$$

so that the bulk part of $\Psi$ is pure gauge and only (the odd part of) its leading term $\bar{\Psi}$ in the asymptotic expansion is truly dynamical (4.10). One can introduce a Lagrange multiplier $\lambda$ for that constraint, so that an alternative action is

$$
\begin{aligned}
S_{H}\left[A_{i}, \pi^{i}, \Psi, \pi_{\Psi} ; A_{t}, \lambda\right]= & \int d t\left\{\int d^{3} x \pi^{i} \partial_{t} A_{i}+\pi_{\Psi} \partial_{t} \Psi-\oint d^{2} x \sqrt{\bar{\gamma}} \bar{A}_{r} \partial_{t} \bar{\Psi}\right. \\
& \left.-\int d^{3} x\left(\frac{1}{2 \sqrt{g}} \pi^{i} \pi_{i}+\frac{\sqrt{g}}{4} F^{i j} F_{i j}\right)-\int d^{3} x\left(\lambda \pi_{\Psi}+A_{t} \mathcal{G}\right)\right\} .
\end{aligned}
$$

In order to avoid a logarithmic divergence in the kinetic term $\int d^{3} x \pi_{\Psi} \partial_{t} \Psi$, we impose that the conjugate momentum $\pi_{\Psi}$ - a density of weight one - , behaves asymptotically in spherical coordinates as

$$
\pi_{\Psi}=\frac{1}{r} \pi_{\Psi}^{(1)}+o\left(r^{-1}\right)
$$

This behaviour does not remove physical solutions since $\pi_{\Psi}=0$ on-shell.

Once the bulk piece is included, the above shifts of $\Psi$ (with the obvious extension in the bulk of the $\bar{\mu}$-transformation, and assuming that $\bar{\mu}$ is field-independent) are generated by the canonical generator

$$
G_{\mu}\left[A_{i}, \Psi, \pi^{i}, \pi_{\Psi}\right]=\int d^{3} x \mu \pi_{\Psi}-\oint d^{2} x \sqrt{\bar{\gamma}} \bar{\mu} \bar{A}_{r}
$$

where the boundary term insures that it is an allowed functional. The generator $G_{\mu}$ reduces to the boundary term when the constraint $\pi_{\Psi} \approx 0$ holds. This conserved boundary term does not generically vanish for odd $\bar{\mu}$ 's and therefore, it indeed defines then a proper gauge transformation in agreement with what was observed before. Contrary to the usual framework, the algebraic constraint $\pi_{\Psi}$ can thus generate improper gauge transformations with non-vanishing charges. This is because the symplectic form involves a non-trivial surface contribution. More information on the canonical formalism with surface-term contributions to the symplectic structure is given in appendix A.

The addition of the surface degree of freedom and the companion boundary modification of the symplectic form lead also to the satisfactory situation that the conserved quantities $-\oint d^{2} x \sqrt{\bar{\gamma}} \bar{\mu} \bar{A}_{r}$ appear as Noether charges for definite, non trivial symmetries. 
Without this surface degree of freedom, the conserved charge $\bar{A}_{r}$ would appear as a conserved quantity that generates no transformation.

To summarize, the extended formulation contains two first-class constraints $\pi_{\Psi} \approx 0$ and $\mathcal{G} \approx 0$ that generate the two independent gauge transformations described above:

$$
\delta_{\mu, \epsilon} \Psi=\mu, \quad \delta_{\mu, \epsilon} A_{i}=\partial_{i} \epsilon, \quad \delta_{\mu, \epsilon} \pi^{i}=0, \quad \delta_{\mu, \epsilon} \pi_{\Psi}=0
$$

where the gauge parameters have the asymptotic behaviour

$$
\mu=\frac{1}{r} \bar{\mu}\left(x^{A}\right)+\frac{1}{r^{2}} \mu^{(1)}\left(x^{A}\right)+o\left(r^{-2}\right), \quad \epsilon=\bar{\epsilon}\left(x^{A}\right)+\frac{1}{r} \epsilon^{(1)}\left(x^{A}\right)+o\left(r^{-1}\right) .
$$

The gauge transformations are improper when the leading terms $\bar{\mu}$, respectively $\bar{\epsilon}$, have an odd, respectively even, part. There is thus a wealth of two functions of the angles, one odd and the other even, of improper gauge transformations. Assuming that $\bar{\mu}$ and $\bar{\epsilon}$ are field-independent, we can write the total generator:

$$
G_{\mu, \epsilon}\left[A_{i}, \Psi, \pi^{i}, \pi_{\Psi}\right]=\int d^{3} x\left(\mu \pi_{\Psi}+\epsilon \mathcal{G}\right)+\oint d^{2} x\left(\overline{\epsilon \pi}^{r}-\sqrt{\bar{\gamma}} \bar{\mu} \bar{A}_{r}\right)
$$

and easily check that it is conserved

$$
\left\{G_{\mu, \epsilon}, H\right\}=\int d^{3} x \pi^{i} \partial_{i} \mu \approx 0 .
$$

To complete the formulation, we need to give boundary conditions on the Lagrangian multiplier $\lambda$. Just as we did for $A_{t}$, we allow it to behave as a gauge transformation asymptotically,

$$
\lambda=\frac{\bar{\lambda}}{r}+\frac{\lambda^{(1)}}{r^{2}}+o\left(r^{-2}\right)
$$

When $\bar{\lambda}\left(-x^{A}\right)=-\bar{\lambda}\left(x^{A}\right)$, the gauge transformation is improper.

We have written the action principle assuming that the electric charge $\oint d^{2} x \bar{\pi}^{r}$ was fixed, allowing the asymptotic value of the "conjugate chemical potential" $\bar{A}_{t}$ to vary. Alternatively, one can fix $\bar{A}_{t}$ and vary the electric charge, in which case one needs to add the surface term $\int d t \oint d^{2} x \bar{A}_{t} \bar{\pi}^{r}$ at infinity. Similarly we have assumed that the other charge $\oint d^{2} x \bar{A}_{r}$ was fixed since otherwise one gets a clash between the equations of motion obtained by varying $\pi_{\Psi}\left(\partial_{t} \Psi=\lambda\right)$ and $\bar{A}_{r}\left(\partial_{t} \bar{\Psi}=0\right)$, when $\lambda$ involves a transformation at infinity. In fact, the leading term $\bar{\lambda}$ of the Lagrange multiplier acts as chemical potential for the charge $\oint d^{2} x \bar{A}_{r}$. If one adds to the action the surface term $\int d t \oint d^{2} x \bar{\lambda} \bar{A}_{r}$, one goes to the corresponding "grand canonical ensemble" and the charge $\oint d^{2} x \bar{A}_{r}$ can now be varied while keeping $\bar{\lambda}$ fixed.

Finally, we note that there is no particular simplification if $\bar{A}_{A}=\partial_{A} \bar{\Phi}$. The only formula that is affected is (4.9), which becomes $\delta_{b} \bar{\Psi}=\bar{D}^{A}\left(b \partial_{A} \bar{\Phi}\right)+b \bar{A}_{r}$.

\subsection{Poincaré charges}

With the boundary modification of the symplectic charges, all Poincaré transformations are canonical transformations with a well-defined generator. We first consider the kinematical transformations, i.e., spatial translations and rotations. 
As we already stressed before, transformations of the fields under a symmetry are defined up to a gauge transformation in any gauge theory. For spatial translations and rotations, we adjust the gauge transformation in such a way that the action of these spatial symmetries on the fields is the ordinary Lie derivative, i.e.,

$$
\delta_{\xi^{k}} A_{i}=\mathcal{L}_{\xi^{k}} A_{i}, \quad \delta_{\xi^{k}} \Psi=\mathcal{L}_{\xi^{k}} \Psi
$$

where $\Psi$ is a spatial scalar so that $\mathcal{L}_{\xi} \Psi=\xi^{k} \partial_{k} \Psi$ and where the spatial vector $\xi^{k}$ is given by (2.19). This choice, which is somewhat arbitrary, leads to a simple algebra of the charges. Spatial translations and rotations are then generated by

$$
\begin{aligned}
P_{0, \xi^{i}} & =\int d^{3} x\left(\pi^{i} \mathcal{L}_{\xi^{k}} A_{i}+\pi_{\Psi} \mathcal{L}_{\xi^{k}} \Psi\right)+\oint d^{2} x \sqrt{\bar{\gamma}} \bar{\Psi} Y^{A} \partial_{A} \bar{A}_{r} \\
& =\int d^{3} x \xi^{i}\left(F_{i j} \pi^{j}-A_{i} \partial_{j} \pi^{j}+\pi_{\Psi} \partial_{i} \Psi\right)+\oint d^{2} x Y^{A}\left(\bar{A}_{A} \bar{\pi}^{r}+\sqrt{\bar{\gamma}} \bar{\Psi} \partial_{A} \bar{A}_{r}\right) .
\end{aligned}
$$

The boundary term in the expression of the rotation charges (angular momentum) is essential with the twisted boundary conditions in order to have an allowed functional.

The generator of the boosts and time translation is more complicated. We have worked out in subsection 4.2 the transformation rules of $A_{i}$ and $\bar{\Psi}$ under boosts, equations (4.11) and (4.9), respectively. Noting that $\bar{D}^{A}\left(b A_{A}\right)+b \bar{A}_{r}$ is the leading term in the asymptotic value of $\nabla^{i}\left(\xi A_{i}\right)$ with $\xi=b r$, we can extend the transformation law of $\bar{\Psi}$ into the bulk with

$$
\delta_{b} \Psi=\nabla^{i}\left(\xi A_{i}\right)
$$

The boosts are then generated as

$$
\begin{aligned}
P_{\xi, 0}= & \int d^{3} x \xi\left(-\Psi \partial_{i} \pi^{i}-A_{i} \nabla^{i} \pi_{\Psi}+\frac{1}{2 \sqrt{g}} \pi_{i} \pi^{i}+\frac{\sqrt{g}}{4} F_{i j} F^{i j}\right) \\
& +\oint d^{2} x b\left(\bar{\Psi} \bar{\pi}^{r}+\sqrt{\bar{\gamma}} \bar{A}_{A} \bar{D}^{A} \bar{A}_{r}\right) .
\end{aligned}
$$

We take the same formal expression for the generator of time translations where the parameter $\xi$ is given by $\xi=T$ with $\partial_{A} T=0$. This amounts again to a specific choice of the improper gauge transformation included in what is meant by a "time translation" and is again a matter of choice. Our choice leads to a simple algebra. The generator (4.30) is thus generally valid for

$$
\xi=b\left(x^{A}\right) r+T, \quad D_{A} D_{B} b+\bar{\gamma}_{A B} b=0, \quad \partial_{A} T=0
$$

While the surface term is mandatory for the boosts in order to have a well-defined generator, it vanishes for time translations. It should be stressed that the logarithmic divergence in the bulk integral of $P_{\xi, \xi^{i}}$, potentially present for rotations and boosts, cancels due to our asymptotic fall-off. 
The total generator of Poincaré transformations can be written in terms of local diffeomorphisms generators in the following way

$$
\begin{aligned}
P_{\xi, \xi^{i}} & =\int d^{3} x\left(\xi \mathcal{H}^{\mathrm{EM}}+\xi^{i} \mathcal{H}_{i}^{\mathrm{EM}}\right)+\mathcal{B}_{\left(\xi, \xi^{i}\right)}^{\mathrm{EM}}, \\
\mathcal{H}^{\mathrm{EM}} & =-\Psi \partial_{i} \pi^{i}-A_{i} \nabla^{i} \pi_{\Psi}+\frac{1}{2 \sqrt{g}} \pi_{i} \pi^{i}+\frac{\sqrt{g}}{4} F_{i j} F^{i j}, \\
\mathcal{H}_{i}^{\mathrm{EM}} & =F_{i j} \pi^{j}-A_{i} \partial_{j} \pi^{j}+\pi_{\Psi} \partial_{i} \Psi, \\
\mathcal{B}_{\xi, \xi^{i}}^{\mathrm{EM}} & =\oint d^{2} x\left(b\left(\bar{\Psi} \bar{\pi}^{r}+\sqrt{\bar{\gamma}} \bar{A}_{A} \bar{D}^{A} A_{r}\right)+Y^{A}\left(\bar{A}_{A} \bar{\pi}^{r}+\sqrt{\bar{\gamma}} \bar{\Psi} \partial_{A} \bar{A}_{r}\right)\right) .
\end{aligned}
$$

One can easily compute the algebra of the various generators. One finds:

$$
\begin{array}{rlrl}
\left\{P_{\xi_{1}, \xi_{1}^{i}}, P_{\xi_{2}, \xi_{2}^{i}}\right\} & =P_{\widehat{\xi}, \widehat{\xi}^{i}}, & & \\
\left\{G_{\mu, \epsilon}, P_{\xi, \xi^{i}}\right\} & =G_{\widehat{\mu}, \widehat{\epsilon}}, & & \left\{G_{\mu_{1}, \epsilon_{1}}, G_{\mu_{2}, \epsilon_{2}}\right\}=0, \\
\widehat{\xi} & =\xi_{1}^{i} \partial_{i} \xi_{2}-\xi_{2}^{i} \partial_{i} \xi_{1}, & \widehat{\xi}^{i}=\xi_{1}^{j} \partial_{j} \xi_{2}^{i}-\xi_{2}^{j} \partial_{j} \xi_{1}^{i}+g^{i j}\left(\xi_{1} \partial_{j} \xi_{2}-\xi_{1} \partial_{j} \xi_{2}\right), \\
\widehat{\mu} & =\nabla^{i}\left(\xi \partial_{i} \epsilon\right)-\xi^{i} \partial_{i} \mu, & \widehat{\epsilon}=\xi \mu-\xi^{i} \partial_{i} \epsilon . &
\end{array}
$$

It follows from these equations that the algebra of the symmetries is a semi-direct sum of the Poincaré algebra and the abelian algebra parametrized by $\bar{\mu}$ and $\bar{\epsilon}$. The action of the Poincaré subalgebra characterising this semi-direct sum is easily obtained from (4.39):

$$
\delta_{(Y, b, T, W)} \bar{\mu}=Y^{A} \partial_{A} \bar{\mu}-\bar{D}_{A}\left(b \bar{D}^{A} \bar{\epsilon}\right), \quad \delta_{(Y, b, T, W)} \bar{\epsilon}=Y^{A} \partial_{A} \bar{\epsilon}-b \bar{\mu} .
$$

If we had chosen a different improper gauge transformation to accompany the spacetime transformations, the bracket $\left\{P_{\xi_{1}, \xi_{1}^{i}}, P_{\xi_{2}, \xi_{2}^{i}}\right\} \approx P_{\widehat{\xi}, \widehat{\xi}^{i}}$ would have generically been modified by terms containing $G$.

In appendix B, we relate our study to the Lagrangian analysis in hyperbolic slicings, and in appendix $\mathrm{C}$, we prove that the above algebra agrees with the one obtained at null infinity. In particular, we show how the even and odd gauge functions $\epsilon$ and $\mu$ combine to form the full angle-dependent $u(1)$ transformations seen at null infinity. This analysis is very close to the corresponding one for gravity [24,38].

\section{Asymptotic analysis: II. Magnetic charges}

The twisted parity conditions that we just investigated lead to a perfectly consistent picture and a well-defined Hamiltonian formulation. They suffer, however, from two drawbacks. First, they do not allow for the presence of magnetic sources. Second, they generically lead to divergences in the magnetic field as one approaches either the past of future null infinity or the future of past null infinity. This is shown in appendix B. To avoid these singularities, one must impose the extra condition that the even parts of the angular components of the electric field and of $F_{A B}$ vanish.

We shall for these reasons turn to the boundary conditions (3.6) and (3.8) which do not suffer from these drawbacks: they cover magnetic sources as we indicated previously, and are free from singularities as one tends to null infinity. 
It turns out that the asymptotic analysis of these boundary conditions is straightforward. The reason is that the modification of the symplectic term and the addition of non-trivial surface terms to the Poincaré generators is forced by the odd part $\partial_{A} \Phi$ of the angular component $A_{A}$ of the vector potential. The even part drops out in those considerations and raises no extra complication. All the difficulties have thus been already treated in the previous section.

The action takes thus again the form

$$
\begin{aligned}
S_{H}\left[A_{i}, \pi^{i}, \Psi, \pi_{\Psi} ; A_{t}, \lambda\right]= & \int d t\left\{\int d^{3} x \pi^{i} \partial_{t} A_{i}+\pi_{\Psi} \partial_{t} \Psi-\oint d^{2} x \sqrt{\bar{\gamma}} \bar{A}_{r} \partial_{t} \bar{\Psi}\right. \\
& \left.-\int d^{3} x\left(\frac{1}{2 \sqrt{g}} \pi^{i} \pi_{i}+\frac{\sqrt{g}}{4} F^{i j} F_{i j}\right)-\int d^{3} x\left(\lambda \pi_{\Psi}+A_{t} \mathcal{G}\right)\right\}
\end{aligned}
$$

and the Poincaré generators are

$$
\begin{aligned}
P_{\xi, \xi^{i}} & =\int d^{3} x\left(\xi \mathcal{H}^{\mathrm{EM}}+\xi^{i} \mathcal{H}_{i}^{\mathrm{EM}}\right)+\mathcal{B}_{\left(\xi, \xi^{i}\right)}^{\mathrm{EM}}, \\
\mathcal{H}^{\mathrm{EM}} & =-\Psi \partial_{i} \pi^{i}-A_{i} \nabla^{i} \pi_{\Psi}+\frac{1}{2 \sqrt{g}} \pi_{i} \pi^{i}+\frac{\sqrt{g}}{4} F_{i j} F^{i j}, \\
\mathcal{H}_{i}^{\mathrm{EM}} & =F_{i j} \pi^{j}-A_{i} \partial_{j} \pi^{j}+\pi_{\Psi} \partial_{i} \Psi \\
\mathcal{B}_{\xi, \xi^{i}}^{\mathrm{EM}} & =\oint d^{2} x\left(b\left(\bar{\Psi} \bar{\pi}^{r}+\sqrt{\bar{\gamma}} \bar{A}_{A} \bar{D}^{A} A_{r}\right)+Y^{A}\left(\bar{A}_{A} \bar{\pi}^{r}+\sqrt{\bar{\gamma}} \bar{\Psi} \partial_{A} \bar{A}_{r}\right)\right) .
\end{aligned}
$$

The logarithmic divergences in the bulk integrals are absent because the leading $O\left(r^{-1}\right)$ term in the integrand of the boost and rotation generators is odd and thus integrates to zero over the 2 -sphere. One may also replace $\bar{A}_{A}$ by $\partial_{A} \bar{\Phi}$ in the boundary terms, because $\bar{A}_{A}^{\text {even }}$ simply drops out. All the subtle features are introduced by the odd component of $\bar{A}_{A}$ studied above.

The theory possesses the same improper gauge transformations as above generated by

$$
G_{\mu, \epsilon}\left[A_{i}, \Psi, \pi^{i}, \pi_{\Psi}\right]=\int d^{3} x\left(\mu \pi_{\Psi}+\epsilon \mathcal{G}\right)+\oint d^{2} x\left(\bar{\epsilon} \bar{\pi}^{r}-\sqrt{\bar{\gamma}} \bar{\mu} \bar{A}_{r}\right) .
$$

These transformations act only on the $\partial_{A} \bar{\Phi}$-part of $\bar{A}_{A}$ and on $\bar{\psi}$ so that here again, all the conceptual points have been made in the previous section.

This ends the discussion of the general case.

\section{Conclusions}

In this paper, we have proposed new boundary conditions for electromagnetism at spatial infinity. These are (2.12), (2.13), (3.3), (3.6), (3.8), (4.10) and (4.19). These boundary conditions include magnetic sources and fulfill the consistency requirements listed in the introduction. They are invariant under an infinite set of non trivial asymptotic symmetries, labeled by one function on the two-sphere. These angle-dependent $u(1)$ transformations have generically non-vanishing charges and their generator is given by (5.6). The way the even component $(\epsilon)$ and the odd component $(\mu)$ of the symmetry parameters combine 
as compared with the analysis at null infinity is somewhat subtle and worked out in appendix C. We have also worked out the canonical generators of the Poincaré group, which involves an extra surface degree of freedom (eq. (5.2), and computed their algebra with the asymptotic infinite angle-dependent $u(1)$ symmetry (eq. (4.36)-(4.39)). Note that if the charge is not zero, Lorentz invariance is broken since the angle-dependent conserved quantity $G_{\mu, \epsilon}$ transforms non trivially under rotations and boosts, as clearly exhibited by (4.40).

A crucial element in the emergence of the non trivial symmetry at spatial infinity is the twist in the parity conditions on the angular components of the vector potential as compared with the conditions of [13]. This twist is the electromagnetic analog of what was done in [24] for pure gravity. However, as we have shown here, it is sufficient for the twist to be given by an improper gauge transformation. This restricted class of twists enables one to include magnetic monopoles. The same can be done for gravity in order to encompass the Taub-NUT solution, as it will be shown elsewhere.

We stress that the parity conditions given here on the angular components of the potential are not a restriction in the sense that they do not exclude the standard solutions relevant for the usual asymptotic analysis. If they were imposed on the next order, they might of course be a restriction, but they are here conditions on an order that is actually zero for the Liénard-Wichiert potential that plays such an important role in the analysis of the fields at null infinity. We have in fact shown that our results are in agreement with those obtained at null infinity. The non trivial symmetries visible at null infinity do have an expression at spatial infinity and turn out to be also asymptotic symmetries with charges that reduce to surface terms at null infinity ("improper gauge transformations"), with no bulk contribution. Relaxing the parity conditions does not seem necessary from the point of view of known solutions and furthermore, could lead to undesirable divergences in the symplectic structure.

Our work also shows an interesting interplay between the radial components of the fields and their angular components. The radial components, associated with the Coulomb aspect of the electromagnetic field, are subject to parity conditions that reflect what is left of spherical symmetry in a moving frame. At the same time, they act as generators of the infinite-dimensional symmetry on the angular components and dictate what is a proper gauge symmetry and what is an improper one. To allow for the improper ones, the angular components must have a parity-twisted piece with respect to the radial components.

There is a clear difference between electromagnetism and gravity in the asymptotic treatment given here. While the twisted parity conditions are sufficient in the gravity case to ensure a consistent formulation without modification of the symplectic structure, an extra feature appears in the case of electromagnetism. It is that the symplectic structure must be modified by a boundary contribution (as observed first in [11]), and that surface degrees of freedom must be explicitly introduced in a gauge-fixing free approach. It is thanks to this extra structure that the full angle-dependent algebra (and not just that of the even functions) emerge at infinity.

In the case of gravity, the condition that the mixed component $h_{r A}$ of the spatial metric should vanish to leading order was imposed in [24]. If it is not satisfied, the boost charges are not integrable. The treatment of the electromagnetic field given here suggests 
that one might avoid the condition $\bar{h}_{r A}=0$ in gravity by introducing surface degrees of freedom (which could be related to the lapse and the shift by gauge-fixing) and modifying the symplectic structure by a surface term. This might lead to an enlargement of the asymptotic symmetry algebra. We recall in this context that super-rotations [39-43] were not included in the approach of [24]. Perhaps this might open the way to their satisfactory inclusion. Similarly it would be interesting to extend the analysis to the Yang-Mills case, as well as to supergravity where one would have to work out the impact of the twist in parity conditions on the spinors. It is hoped to return to all these questions in the future.

\section{Acknowledgments}

$\mathrm{MH}$ is grateful to the Institute for Advanced Study (Princeton) for kind hospitality while this work was completed. This research was partially supported by the ERC Advanced Grant "High-Spin-Grav", by FNRS-Belgium (convention FRFC PDR T.1025.14 and convention IISN 4.4503.15) and by the "Communauté Française de Belgique" through the ARC program.

\section{A Poisson structure}

We extend in this appendix the work of [14] and [44] to the case when the symplectic form is not the standard one but is modified by a boundary term. This extension relies on wellestablished results of the hamiltonian formalism, as described e.g. in the monograph [45].

In the following, we will collectively denote the fields by $z^{A}$ and we will restrict our analysis to symplectic structures $\Omega$ of the form

$$
\Omega\left[z ; d_{V} z, d_{V} z\right]=\int \frac{1}{2} \sigma_{A B} d_{V} z^{A} d_{V} z^{B} d^{n} x+\omega\left[z ; d_{V} z, d_{V} z\right]
$$

where $\omega$ is a boundary term such that $d_{V} \omega=0$. We will also assume the matrix $\sigma_{A B}=-\sigma_{B A}$ to be constant and invertible $\sigma^{A B} \sigma_{B C}=\delta_{C}^{A}$.

Let us consider a vertical vector field $\delta_{F} z^{A}$ preserving the asymptotic conditions. It is a hamiltonian vector field iff

$$
F\left[z^{A}\right]=\int f(z) d^{n} x+\oint f_{b}(z) d^{n-1} x \quad \text { s.t. } \quad d_{V} F=-i_{F} \Omega\left[z ; d_{V} z, d_{V} z\right]
$$

where $\Omega$ is a closed vertical 2 -form. We will call allowed functionals $F$ functionals that are associated to a hamiltonian vector through (A.2). As in the case of Regge-Teitelboim, this prescription fixes the boundary term of allowed functionals up to a constant.

Considering two allowed functionals $F_{1}$ and $F_{2}$, we can define their Poisson bracket as

$$
\left\{F_{1}, F_{2}\right\}=i_{1} i_{2} \Omega=\int \frac{\delta F_{1}}{\delta z^{A}} \sigma^{A B} \frac{\delta F_{2}}{\delta z^{B}} d^{n} x+i_{1} i_{2} \omega .
$$

In this case, we see that the usual result given by the bulk term is modified by a boundary term. By construction, the Poisson bracket is antisymmetric and we have the following property

$$
\left\{F_{1}, F_{2}\right\}=-i_{2} i_{1} \Omega=i_{2} d_{V} F_{1}=\delta_{2} F_{1}
$$


where $\delta_{2} z^{A}$ is the variation associated to $F_{2}$. Using standard techniques, we can then prove the two following results that make this Poisson bracket well-defined.

Theorem A.1 The Poisson bracket of two allowed functionals $F_{1}$ and $F_{2}$ is an allowed functional with

$$
d_{V}\left\{F_{1}, F_{2}\right\}=-i_{[2,1]} \Omega
$$

Theorem A.2 The Poisson bracket satisfies Jacobi identity:

$$
\left\{\left\{F_{1}, F_{2}\right\}, F_{3}\right\}+\text { cyclic }=0 .
$$

The demonstration of these theorems follow usual lines of symplectic geometry and is left to the reader.

In the particular case where the boundary term of the symplectic structure is absent, $\omega=0$, the allowed functionals $F$ are those with a variation of the form

$$
d_{V} F=\int \frac{\delta F}{\delta z^{A}} d_{V} z^{A} d^{n} x-i_{F} \omega=\int \frac{\delta F}{\delta z^{A}} d_{V} z^{A} d^{n} x
$$

without boundary term. This is the prescription of [14].

\section{B Electromagnetism in hyperbolic slicings}

\section{B.1 Connection with previous work}

The connection between spatial infinity and null infinity is most conveniently worked out in hyperbolic coordinates [46-49],

$$
\begin{aligned}
& g_{\mu \nu} d x^{\mu} d x^{\nu}=d \eta^{2}+\eta^{2} h_{a b} d x^{a} d x^{b} \\
& h_{a b} d x^{a} d x^{b}=\frac{-1}{\left(1-s^{2}\right)^{2}} d s^{2}+\frac{1}{1-s^{2}} \bar{\gamma}_{A B} d x^{A} d x^{B}
\end{aligned}
$$

where $\bar{\gamma}_{A B}$ is the metric on the 2 -sphere. The radial coordinate $\eta$ and the hyperbolic time $s$ are related to the usual time $t$ and equal time radial distance $r$ by

$$
\eta=\sqrt{-t^{2}+r^{2}}, \quad s=\frac{t}{r} .
$$

These coordinates only cover the part of space-time where $r \geq|t|$ which implies $-1 \leq s \leq 1$. Tensors appearing in the $\eta$ expansions are defined on the 3 dimensional unit hyperboloid $\mathcal{H}$ with metric $h_{a b}$ coordinates $x^{a}=\left(s, x^{A}\right)$ and covariant derivative $\mathcal{D}_{a}$. A translation in the hyperbolic time $s$ involves asymptotic boosts at infinity.

The analysis of electromagnetism in hyperbolic coordinates was performed in [11], in which the need to modify the symplectic form by a boundary term was first pointed out. In this appendix, we compare our findings in standard coordinates with the results of [11]. In the next appendix, we use the hyperbolic coordinates to relate the asymptotic symmetry group at spatial infinity with the asymptotic symmetry group at null infinity. 
Reference [11] assumes the following fall-offs for the electromagnetic potential:

$$
A_{a}=\bar{A}_{a}+A_{a}^{(1)} \frac{1}{\eta}+o\left(\eta^{-1}\right), \quad A_{\eta}=\bar{A}_{\eta} \frac{1}{\eta}+A_{\eta}^{(1)} \frac{1}{\eta^{2}}+o\left(\eta^{-2}\right)
$$

With this asymptotic behaviour, the usual bulk action of electromagnetism is not welldefined. A general variation of the action leads indeed to:

$$
\begin{aligned}
\delta \int d^{4} x \frac{-\sqrt{-g}}{4} F_{\mu \nu} F^{\mu \nu}= & \int d^{4} x \sqrt{-g} \nabla_{\mu} F^{\mu \nu} \delta A_{\nu} \\
& +\left.\int d \eta \oint d^{2} x \sqrt{-g} F^{s \nu} \delta A_{\nu}\right|_{s_{0}} ^{s_{1}}+\int_{\mathcal{H}} d^{3} x \sqrt{-h} \mathcal{D}^{a} \bar{A}_{\eta} \delta \bar{A}_{a}
\end{aligned}
$$

where $\nabla_{\mu}$ is the covariant derivative associated to the background metric (B.1). There is a boundary term at the spatial boundary $\mathcal{H}$.

A solution for removing this unwanted term that allows one to keep a non-trivial asymptotic symmetry algebra without having an impact on the solution space is given in [11]. One adds the following boundary term to the action:

$$
S\left[A_{\mu}\right]=\int d^{4} x \frac{-\sqrt{-g}}{4} F_{\mu \nu} F^{\mu \nu}+\int_{\mathcal{H}} d^{3} x \sqrt{-h} \bar{A}_{\eta}\left(\mathcal{D}^{a} \bar{A}_{a}+\bar{A}_{\eta}\right)
$$

Here, we have used a boundary term quadratic in $\bar{A}_{\eta}$ but a more general function of $\bar{A}_{\eta}$ could have been used.

A variation of the action now gives

$$
\begin{aligned}
\delta S= & \int d^{4} x \sqrt{-g} \nabla_{\mu} F^{\mu \nu} \delta A_{\nu}+\int_{\mathcal{H}} d^{3} x \sqrt{-h}\left(\mathcal{D}^{a} \bar{A}_{a}+2 \bar{A}_{\eta}\right) \delta \bar{A}_{\eta} \\
& +\left[\int d \eta \oint d^{2} x \sqrt{-g} F^{s \nu} \delta A_{\nu}+\oint d^{2} x \sqrt{-h} h^{s a} \bar{A}_{\eta} \delta \bar{A}_{a}\right]_{s_{0}}^{s_{1}} .
\end{aligned}
$$

The choice of boundary term added to the action leads to an extra equation of motion on the boundary:

$$
\mathcal{D}^{a} \bar{A}_{a}+2 \bar{A}_{\eta}=0
$$

This is the leading term of Lorenz gauge condition:

$$
\nabla^{\mu} A_{\mu}=\frac{1}{\eta^{2}}\left(\mathcal{D}^{a} \bar{A}_{a}+2 \bar{A}_{\eta}\right)+o\left(\eta^{-2}\right)
$$

The extra EOM (B.8) is compatible with all bulk EOM and can be interpreted as an asymptotic gauge fixing. This condition is a central element of the analysis of [11] where it is imposed as an extra asymptotic condition (see also [50]). We have just shown that it consistently follows in fact dynamically from the action. The gauge transformations preserving the action are generated by gauge parameter of the form

$$
\lambda\left(\eta, x^{a}\right)=\bar{\lambda}\left(x^{a}\right)+\lambda^{(1)}\left(x^{a}\right) \frac{1}{\eta}+o\left(\eta^{-1}\right), \quad \mathcal{D}_{a} \mathcal{D}^{a} \bar{\lambda}=0
$$


Here and in the next appendix, we used the notation $\lambda$ for the gauge parameter in order to make the link with [11]. It is not related to the extra Lagrange multiplier we added in section 4.4.

Our analysis of the gauge symmetries and associated charges, translated in hyperbolic coordinates, reduces to that of [11] if one imposes the gauge condition $A_{t}=\Psi$, which is admissible if the constant part in the asymptotic expansion of $A_{t}$ is absent (otherwise, one must impose $A_{t}-\bar{A}_{t}=\Psi$ ). Since the Lagrange multiplier $A_{t}$ transforms with the time derivative of the gauge parameter $\epsilon$, this gauge condition relates $\partial_{t} \epsilon$ to $\mu$. Note also that $s$-translations in hyperbolic coordinates involve asymptotic boosts. It does therefore not come as a surprise that the symplectic form problem encountered in our treatment when handling Lorentz boosts appear here already at the level of $(s-)$ time translations.

The action (B.6) suffers from a second problem: the associated symplectic structure $\Omega$ is divergent. This problem is solved in the same way as in the core of our paper by adding parity conditions on gauge invariant combinations of the asymptotic fields. The discussion proceeds along parallel tracks and will thus not be repeated here.

\section{B.2 Asymptotic behaviour of the fields as one goes to null infinity}

To end this appendix, we will make the link between the asymptotic fields in hyperbolic coordinates and physical quantities usually defined at null infinity. The equations of motion derived from action (B.6) imply

$$
-\left(1-s^{2}\right) \partial_{s}^{2} \bar{A}_{\eta}+\bar{D}_{A} \bar{D}^{A} \bar{A}_{\eta}=0, \quad \bar{D}^{B} \bar{F}_{B A}-\partial_{s}\left(\left(1-s^{2}\right) \bar{F}_{s A}\right)=0
$$

where $\bar{F}_{a b}=\partial_{a} \bar{A}_{b}-\partial_{b} \bar{A}_{a}$ is the leading term of the field strength $F_{a b}=\bar{F}_{a b}+O\left(\eta^{-1}\right)$. These two equations control the asymptotic behaviour of the radial part of the electric and magnetic field:

$$
\begin{array}{ll}
E^{\eta}\left(\eta, x^{a}\right)=\sqrt{-g} F^{s \eta}=\bar{E}^{\eta}\left(x^{a}\right)+O\left(\eta^{-1}\right), & \bar{E}^{\eta}=-\sqrt{\bar{\gamma}} \partial_{s} \bar{A}_{\eta}, \\
B^{\eta}\left(\eta, x^{a}\right)=\epsilon^{s \eta A B} F_{A B}=\bar{B}^{\eta}\left(x^{a}\right)+O\left(\eta^{-1}\right), & \bar{B}^{\eta}=-\epsilon^{A B} \bar{F}_{A B} .
\end{array}
$$

We have denoted by $\epsilon^{\alpha \beta \gamma \delta}$ and $\epsilon^{A B}$ the anti-symmetric densities with $\epsilon^{s \eta \theta \phi}=-1=-\epsilon^{\theta \phi}$. Using Bianchi's identity, one can easily show that the leading terms of both $E^{\eta}$ and $B^{\eta}$ satisfy the following equation:

$$
-\partial_{s}\left(\left(1-s^{2}\right) \partial_{s} \Xi\right)+\bar{D}_{A} \bar{D}^{A} \Xi=0, \quad \Xi=\bar{E}^{\eta}, \bar{B}^{\eta} .
$$

Using spherical harmonics, its general solution can be written in terms of Legendre polynomials $P_{l}(s)$ and Legendre functions of the second kind $Q_{l}(s)$ which can be expressed in terms of $P_{l}(s)$ as

$$
Q_{l}(s)=P_{l}(s) \frac{1}{2} \log \left(\frac{1+s}{1-s}\right)+\widetilde{Q}_{l}(s)
$$


where $\widetilde{Q}_{l}(s)$ are polynomials. Without taking into account the parity conditions, the corresponding solutions are given as follows

$$
\begin{aligned}
& \bar{E}^{\eta}\left(s, x^{A}\right)=\sqrt{\gamma} \sum_{l, m}\left(E_{l, m}^{P} P_{l}(s)+E_{l, m}^{Q} Q_{l}(s)\right) Y_{l, m}\left(x^{A}\right), \\
& \bar{B}^{\eta}\left(s, x^{A}\right)=\sqrt{\gamma} \sum_{l, m}\left(B_{l, m}^{P} P_{l}(s)+B_{l, m}^{Q} Q_{l}(s)\right) Y_{l, m}\left(x^{A}\right) .
\end{aligned}
$$

Encoding the contribution from electric sources, $\bar{E}^{\eta}$ is always even on the hyperboloid which means that $E_{l, m}^{Q}=0$. This implies that the leading part of the electric field $\bar{E}^{\eta}$ is bounded in the interval $s \in[-1,1]$. Its magnetic counterpart $\bar{B}^{\eta}$ will also be bounded if one imposes parity conditions allowing for magnetic charges, $\bar{B}_{o d d}^{\eta}=0=B_{l, m}^{Q}$, however, it will diverges at $s= \pm 1$ if one imposes twisted boundary conditions, $\bar{B}_{\text {even }}^{\eta}=0=B_{l, m}^{P}$.

As one could expect, the late time behaviour of both quantities is related to their value at null infinity. Unfortunately, the link is not direct as the hyperbolic coordinates used above are not adapted to the description of null infinity. We will instead rely on the methods of [51-53] and introduce a new radial coordinate $\rho=\eta\left(1-s^{2}\right)^{\frac{1}{2}}$. With this, the background metric takes the form

$$
d s^{2}=\frac{1}{\left(1-s^{2}\right)^{2}}\left(\left(1-s^{2}\right) d \rho^{2}+2 s \rho d s d \rho-\rho^{2} d s^{2}+\rho^{2} \gamma_{A B} d x^{A} d x^{B}\right)
$$

In these new coordinates, the rescaled line element $\left(1-s^{2}\right)^{2} d s^{2}$ is continuous in the limits $s \rightarrow \pm 1$. These two hypersurfaces describe past and future null infinity with induced coordinates $\left(\rho, x^{A}\right)$. The limits relevant for Strominger's analysis, namely approaching spatial infinity along future or past null infinity, correspond to taking the following two limits in succession: $s \rightarrow 1$ or $s \rightarrow-1$ and then $\rho \rightarrow \infty$. As the change of coordinates only involves a rescaling of the radial coordinate, the expansion of the fields given in (B.4) behaves nicely. In particular, the leading term of the radial electric and magnetic fields are unchanged

$$
E^{\rho}\left(\rho, x^{a}\right)=\sqrt{-g} F^{s \rho}=\bar{E}^{\eta}\left(x^{a}\right)+O\left(\rho^{-1}\right), \quad B^{\rho}\left(\rho, x^{a}\right)=\epsilon^{s \rho A B} F_{A B}=\bar{B}^{\eta}\left(x^{a}\right)+O\left(\rho^{-1}\right) .
$$

Using the explicit solutions obtained in (B.16), we can now evaluate the value of the electromagnetic field at null infinity. The leading part of the electric field $E^{\rho}$ is finite and its parity properties imply that it will satisfy the antipodal matching conditions of Strominger [2]. However, if one imposes twisted boundary conditions, the magnetic field will in general diverge in the limits $s \rightarrow \pm 1$ which breaks the usual asymptotic conditions of electromagnetism at null infinity.

\section{Explicit large gauge transformations and link with null infinity}

We compare in this appendix the asymptotic symmetry group appearing here at spatial infinity with the asymptotic symmetry group emerging at null infinity. Using hyperbolic 
coordinates to describe spatial infinity, large U(1) gauge transformations are parametrized by gauge parameters of the form

$$
\lambda\left(\eta, x^{a}\right)=\bar{\lambda}\left(x^{a}\right)+\lambda^{(1)}\left(x^{a}\right) \eta^{-1}+o\left(\eta^{-1}\right)
$$

where $\bar{\lambda}$ is an even function defined on the hyperboloid and satisfying

$$
\mathcal{D}_{a} \mathcal{D}^{a} \bar{\lambda}=-\left(1-s^{2}\right)^{2} \partial_{s}^{2} \bar{\lambda}+\left(1-s^{2}\right) \bar{D}_{A} \bar{D}^{A} \bar{\lambda}=0
$$

(see (B.10)). On can check that the odd part of $\bar{\lambda}$ is always a proper gauge transformation due to the parity conditions imposed on $\bar{E}^{\eta}$. The general solution to this equation that is also an even function can be written in terms of spherical harmonics as follows

$$
\bar{\lambda}\left(s, x^{A}\right)=\sum_{l, m} \bar{\lambda}_{l, m} \alpha_{l}(s) Y_{l, m}\left(x^{A}\right), \quad \alpha_{l}=\left(1-s^{2}\right) \partial_{s} Q_{l}(s)
$$

where the functions $Q_{l}(s)$ are Legendre functions of the second kind given in terms of Legendre polynomials $P_{l}(s)$ in equation (B.15). The fact that $P_{l}( \pm 1)=(-1)^{l}$ can be used to determine the asymptotic behaviour of the functions $\alpha_{l}$

$$
\lim _{s \rightarrow \pm 1} \alpha_{l}(s)=\lim _{s \rightarrow \pm 1} P_{l}(s)=( \pm 1)^{l}, \quad \lim _{s \rightarrow \pm 1}\left(1-s^{2}\right) \partial_{s} \alpha_{l}(s)=0 .
$$

The general solution obtained in (C.3) is completely characterised by its value at $s= \pm 1$ :

$$
\lim _{s \rightarrow \pm 1} \bar{\lambda}\left(s, x^{A}\right)=\epsilon^{\mathcal{J}}\left( \pm x^{A}\right)
$$

These two values correspond to the gauge parameters at future and past null infinity. As explained in the previous appendix, in order to show this, we need to introduce a new radial coordinate $\rho=\eta\left(1-s^{2}\right)^{-\frac{1}{2}}$. The gauge parameters induced at future and past null infinity can then be evaluated easily

$$
\left.\lambda\left(\rho, x^{A}\right)\right|_{\mathcal{J}^{ \pm}}=\lim _{s \rightarrow \pm 1} \lambda\left(\rho, x^{a}\right)=\epsilon^{\mathcal{J}}\left( \pm x^{A}\right)+O\left(\rho^{-1}\right)
$$

where sub-leading terms in $\rho$ are present because the gauge in the bulk is not fixed. One can see that the antipodal properties of the gauge parameter obtained in [2] are consequences of the parity conditions associated with the extra equation of motion (B.8).

The link with the parametrization of the $\mathrm{U}(1)$ transformations used in the Hamiltonian formalism in section 4 is made by parametrising the function $\bar{\lambda}$, solution of equation (C.2), in terms of initial conditions given at $s=0$ :

$$
\left.\bar{\lambda}\right|_{s=0}=\bar{\epsilon}\left(x^{A}\right),\left.\quad \partial_{s} \bar{\lambda}\right|_{s=0}=\bar{\mu}\left(x^{A}\right)
$$

Both $\bar{\epsilon}$ and $\bar{\mu}$ are functions on the sphere with a definite parity: $\bar{\epsilon}\left(-x^{A}\right)=\bar{\epsilon}\left(x^{A}\right)$ and $\bar{\mu}\left(-x^{A}\right)=-\bar{\mu}\left(x^{A}\right)$. The explicit change of parametrisation from null infinity $\epsilon^{\mathcal{J}}$ and the 
Hamiltonian formalism at spatial infinity $(\bar{\epsilon}, \bar{\mu})$ can be obtained using the expansion in spherical harmonics and the expressions for the functions $\alpha_{l}$ :

$$
\begin{aligned}
& \epsilon^{\mathcal{J}}=\sum_{l, m} \bar{\lambda}_{l, m} Y_{l, m}\left(x^{A}\right), \\
& \bar{\mu}=\sum_{k} \sum_{m=-2 k-1}^{2 k+1} \bar{\mu}_{2 k+1, m} Y_{2 k+1, m}, \quad \bar{\epsilon}=\sum_{k} \sum_{m=-2 k}^{2 k} \bar{\epsilon}_{2 k, m} Y_{2 k, m}, \\
& \left.\bar{\lambda}_{2 k+1, m} \partial_{s} \alpha_{2 k+1}\right|_{s=0}=\bar{\mu}_{2 k+1, m},\left.\quad \bar{\lambda}_{2 k, m} \alpha_{2 k}\right|_{s=0}=\bar{\epsilon}_{2 k, m} .
\end{aligned}
$$

The first few $\alpha_{l}$ functions can be easily computed

$$
\alpha_{0}=1, \quad \alpha_{1}=s+\frac{1-s^{2}}{2} \log \left(\frac{1+s}{1-s}\right), \quad \alpha_{2}=3 s^{2}-2+\frac{3 s\left(1-s^{2}\right)}{2} \log \left(\frac{1+s}{1-s}\right)
$$

and we can use them to write the first few component of the change of basis:

$$
\bar{\lambda}_{0,0}=\bar{\epsilon}_{0,0}, \quad \bar{\lambda}_{1, m}=\frac{1}{2} \bar{\mu}_{1, m}, \quad \bar{\lambda}_{2, m}=-\frac{1}{2} \bar{\epsilon}_{2, m} .
$$

The action of Lorentz algebra on both parametrizations of the large U(1) gauge transformations can be evaluated using the same strategy. Lorentz algebra on the hyperboloid is generated by

$$
\mathcal{Y}^{s}=-\left(1-s^{2}\right) b, \quad \mathcal{Y}^{A}=Y^{A}-s \bar{D}^{A} b,
$$

and acts on the $\mathrm{U}(1)$ gauge parameter $\bar{\lambda}$ through the Lie derivative. We obtain its action on the gauge parameter at null infinity $\epsilon^{\mathcal{J}}$ by taking the limit $s \rightarrow 1$ :

$$
\delta_{Y, b} \epsilon^{\mathcal{J}}=\lim _{s \rightarrow 1} \delta_{Y, b} \bar{\lambda}=\left(Y^{A}-\bar{D}^{A} b\right) \partial_{A} \epsilon^{\mathcal{J}}
$$

which is the Lorentz action on the gauge parameter at null infinity [3]. The action of Lorentz algebra in the alternative parametrization can be obtained by evaluation of the transformation laws of $\bar{\lambda}$ and $\partial_{s} \bar{\lambda}$ at $s=0$ :

$$
\delta_{Y, b} \bar{\epsilon}=-b \bar{\mu}+Y^{A} \partial_{A} \bar{\epsilon}, \quad \delta_{Y, b} \bar{\mu}=-\bar{D}_{A}\left(b \bar{D}^{A} \epsilon\right)+Y^{A} \partial_{A} \bar{\mu} .
$$

This is the action obtained in section 4 which finishes the proof that the algebra obtained in the hamiltonian formalism is identical to the one obtained at null infinity.

Note that in the null infinity terminology, the asymptotic symmetry group at spatial infinity is the diagonal subgroup of the groups of angle-dependent transformations at future null infinity and past null infinity.

Open Access. This article is distributed under the terms of the Creative Commons Attribution License (CC-BY 4.0), which permits any use, distribution and reproduction in any medium, provided the original author(s) and source are credited. 


\section{References}

[1] A.P. Balachandran and S. Vaidya, Spontaneous Lorentz Violation in Gauge Theories, Eur. Phys. J. Plus 128 (2013) 118 [arXiv:1302.3406] [InSPIRE].

[2] A. Strominger, Asymptotic Symmetries of Yang-Mills Theory, JHEP 07 (2014) 151 [arXiv: 1308.0589] [INSPIRE].

[3] G. Barnich and P.-H. Lambert, Einstein-Yang-Mills theory: Asymptotic symmetries, Phys. Rev. D 88 (2013) 103006 [arXiv:1310.2698] [INSPIRE].

[4] Z. Bern, S. Davies, P. Di Vecchia and J. Nohle, Low-Energy Behavior of Gluons and Gravitons from Gauge Invariance, Phys. Rev. D 90 (2014) 084035 [arXiv:1406.6987] [INSPIRE].

[5] T. He, P. Mitra, A.P. Porfyriadis and A. Strominger, New Symmetries of Massless QED, JHEP 10 (2014) 112 [arXiv: 1407.3789] [InSPIRE].

[6] V. Lysov, S. Pasterski and A. Strominger, Low's Subleading Soft Theorem as a Symmetry of QED, Phys. Rev. Lett. 113 (2014) 111601 [arXiv:1407.3814] [INSPIRE].

[7] D. Kapec, V. Lysov and A. Strominger, Asymptotic Symmetries of Massless QED in Even Dimensions, Adv. Theor. Math. Phys. 21 (2017) 1747 [arXiv:1412.2763] [InSPIRE].

[8] D. Kapec, M. Pate and A. Strominger, New Symmetries of QED, Adv. Theor. Math. Phys. 21 (2017) 1769 [arXiv: 1506.02906] [INSPIRE].

[9] M. Campiglia and A. Laddha, Subleading soft photons and large gauge transformations, JHEP 11 (2016) 012 [arXiv: 1605.09677] [INSPIRE].

[10] E. Conde and P. Mao, Remarks on asymptotic symmetries and the subleading soft photon theorem, Phys. Rev. D 95 (2017) 021701 [arXiv:1605.09731] [INSPIRE].

[11] M. Campiglia and R. Eyheralde, Asymptotic U(1) charges at spatial infinity, JHEP 11 (2017) 168 [arXiv:1703.07884] [INSPIRE].

[12] A. Strominger, Lectures on the Infrared Structure of Gravity and Gauge Theory, arXiv: 1703.05448 [INSPIRE].

[13] M. Henneaux, B. Julia and S. Silva, Noether superpotentials in supergravities, Nucl. Phys. B 563 (1999) 448 [hep-th/9904003] [INSPIRE].

[14] T. Regge and C. Teitelboim, Role of Surface Integrals in the Hamiltonian Formulation of General Relativity, Annals Phys. 88 (1974) 286 [InSPIRE].

[15] R. Benguria, P. Cordero and C. Teitelboim, Aspects of the Hamiltonian Dynamics of Interacting Gravitational Gauge and Higgs Fields with Applications to Spherical Symmetry, Nucl. Phys. B 122 (1977) 61 [InSPIRE].

[16] H. Bondi, M.G.J. van der Burg and A.W.K. Metzner, Gravitational waves in general relativity. 7. Waves from axisymmetric isolated systems, Proc. Roy. Soc. Lond. A 269 (1962) 21 [INSPIRE].

[17] R.K. Sachs, Gravitational waves in general relativity. 8. Waves in asymptotically flat space-times, Proc. Roy. Soc. Lond. A 270 (1962) 103 [InSPIRE].

[18] R. Sachs, Asymptotic symmetries in gravitational theory, Phys. Rev. 128 (1962) 2851 [INSPIRE]. 
[19] E.T. Newman and T.W.J. Unti, Behavior of Asymptotically Flat Empty Spaces, J. Math. Phys. 3 (1962) 891 [INSPIRE].

[20] R. Penrose, Asymptotic properties of fields and space-times, Phys. Rev. Lett. 10 (1963) 66 [INSPIRE].

[21] R. Penrose, Zero rest mass fields including gravitation: Asymptotic behavior, Proc. Roy. Soc. Lond. A 284 (1965) 159 [INSPIRE].

[22] T. Mädler and J. Winicour, Bondi-Sachs Formalism, Scholarpedia 11 (2016) 33528 [arXiv: 1609.01731] [INSPIRE].

[23] F. Alessio and G. Esposito, On the structure and applications of the Bondi-Metzner-Sachs group, Int. J. Geom. Meth. Mod. Phys. 15 (2017) 1830002 [arXiv:1709.05134] [InSPIRE].

[24] M. Henneaux and C. Troessaert, BMS Group at Spatial Infinity: the Hamiltonian (ADM) approach, JHEP 03 (2018) 147 [arXiv:1801.03718] [INSPIRE].

[25] J. Fröhlich, G. Morchio and F. Strocchi, Infrared Problem And Spontaneous Breaking Of The Lorentz Group In Qed, Phys. Lett. B 89 (1979) 61 [InSPIRE].

[26] J. Fröhlich, G. Morchio and F. Strocchi, Charged Sectors and Scattering States in Quantum Electrodynamics, Annals Phys. 119 (1979) 241 [INSPIRE].

[27] A. Herdegen, Long range effects in asymptotic fields and angular momentum of classical field electrodynamics, J. Math. Phys. 36 (1995) 4044 [inSPIRE].

[28] A. Ashtekar, S. Fairhurst and B. Krishnan, Isolated horizons: Hamiltonian evolution and the first law, Phys. Rev. D 62 (2000) 104025 [gr-qc/0005083] [INSPIRE].

[29] J.-L. Gervais, B. Sakita and S. Wadia, The Surface Term in Gauge Theories, Phys. Lett. B 63 (1976) 55 [INSPIRE].

[30] S. Wadia and T. Yoneya, The Role of Surface Variables in the Vacuum Structure of Yang-Mills Theory, Phys. Lett. B 66 (1977) 341 [INSPIRE].

[31] S.R. Wadia, Hamiltonian Formulation of Nonabelian Gauge Theory with Surface Terms: Applications to the Dyon Solution, Phys. Rev. D 15 (1977) 3615 [INSPIRE].

[32] J.-L. Gervais and D. Zwanziger, Derivation From First Principles of the Infrared Structure of Quantum Electrodynamics, Phys. Lett. B 94 (1980) 389 [InSPIRE].

[33] G. Dvali, C. Gomez and N. Wintergerst, Stückelberg Formulation of Holography, Phys. Rev. D 94 (2016) 084051 [arXiv:1511.03525] [INSPIRE].

[34] J.D. Brown and M. Henneaux, Central Charges in the Canonical Realization of Asymptotic Symmetries: An Example from Three-Dimensional Gravity, Commun. Math. Phys. 104 (1986) 207 [INSPIRE].

[35] M. Henneaux, C. Martinez, R. Troncoso and J. Zanelli, Black holes and asymptotics of $2+1$ gravity coupled to a scalar field, Phys. Rev. D 65 (2002) 104007 [hep-th/0201170] [INSPIRE].

[36] M. Henneaux, C. Martinez, R. Troncoso and J. Zanelli, Asymptotically anti-de Sitter spacetimes and scalar fields with a logarithmic branch, Phys. Rev. D 70 (2004) 044034 [hep-th/0404236] [INSPIRE].

[37] M. Henneaux, C. Martinez, R. Troncoso and J. Zanelli, Asymptotic behavior and Hamiltonian analysis of anti-de Sitter gravity coupled to scalar fields, Annals Phys. $\mathbf{3 2 2}$ (2007) 824 [hep-th/0603185] [inSPIRE]. 
[38] C. Troessaert, The BMS4 algebra at spatial infinity, Class. Quant. Grav. 35 (2018) 074003 [arXiv: 1704.06223] [INSPIRE].

[39] T. Banks, A critique of pure string theory: Heterodox opinions of diverse dimensions, hep-th/0306074 [INSPIRE].

[40] G. Barnich and C. Troessaert, Symmetries of asymptotically flat 4 dimensional spacetimes at null infinity revisited, Phys. Rev. Lett. 105 (2010) 111103 [arXiv:0909.2617] [INSPIRE].

[41] G. Barnich and C. Troessaert, Aspects of the BMS/CFT correspondence, JHEP 05 (2010) 062 [arXiv: 1001.1541 ] [inSPIRE].

[42] G. Barnich and C. Troessaert, BMS charge algebra, JHEP 12 (2011) 105 [arXiv:1106.0213] [INSPIRE].

[43] G. Barnich and C. Troessaert, Finite BMS transformations, JHEP 03 (2016) 167 [arXiv: 1601.04090] [INSPIRE].

[44] J.D. Brown and M. Henneaux, On the Poisson Brackets of Differentiable Generators in Classical Field Theory, J. Math. Phys. 27 (1986) 489 [inSPIRE].

[45] V.I. Arnold, Mathematical Methods of Classical Mechanics, Graduate Texts in Mathematics, Springer, New York (1989).

[46] A. Ashtekar and R.O. Hansen, A unified treatment of null and spatial infinity in general relativity. I - Universal structure, asymptotic symmetries and conserved quantities at spatial infinity, J. Math. Phys. 19 (1978) 1542 [InSPIRE].

[47] R. Beig and B. Schmidt, Einstein's equations near spatial infinity, Commun. Math. Phys. 87 (1982) 65 .

[48] R. Beig, Integration Of Einstein's Equations Near Spatial Infinity, Proc. Roy. Soc. Lond. A 391 (1984) 295.

[49] A. Ashtekar and J.D. Romano, Spatial infinity as a boundary of space-time, Class. Quant. Grav. 9 (1992) 1069 [INSPIRE].

[50] H. Afshar, E. Esmaeili and M.M. Sheikh-Jabbari, Asymptotic Symmetries in p-Form Theories, arXiv: 1801.07752 [INSPIRE].

[51] H. Friedrich, Gravitational fields near space-like and null infinity, J. Geom. Phys. 24 (1998) 83.

[52] H. Friedrich and J. Kannar, Bondi type systems near space-like infinity and the calculation of the NP constants, J. Math. Phys. 41 (2000) 2195 [gr-qc/9910077] [INSPIRE].

[53] H. Friedrich and J. Kannar, Calculating asymptotic quantities near space - like and null infinity from Cauchy data, Annalen Phys. 9 (2000) 321 [gr-qc/9911103] [INSPIRE]. 